УДК 517.5

\author{
A. O. Kuryliak, V. L. Tsvigun
}

\title{
WIMAN'S TYPE INEQUALITY FOR MULTIPLE POWER SERIES IN AN UNBOUNDED CYLINDER DOMAIN
}

\begin{abstract}
A. O. Kuryliak, V. L. Tsvigun. Wiman's type inequality for multiple power series in an unbounded cylinder domain, Mat. Stud. 49 (2018), 29-51.

In this paper we prove some analogues of Wiman's inequality for analytic $f(z)$ and random analytic functions $f(z, t)$ on $\mathbb{T}=\mathbb{D}^{l} \times \mathbb{C}^{p-l}, l \in \mathbb{N}, 1 \leq l<p, I=\{1, \ldots, l\}, J=\{l+1, \ldots, p\}$ of the form $f(z)=\sum_{\|n\|=0}^{+\infty} a_{n} z^{n}, f(z, t)=\sum_{\|n\|=0}^{+\infty} a_{n} Z_{n}(t) z^{n}$, respectively. Here $Z=\left(Z_{n}\right)$ is a multiplicative system of random variables on the Steinhaus probability space, uniformly bounded by the number 1. In particular, we prove the following statements: For every $\delta>0$ there exist sets $E_{1}=E_{1}(\delta, f), E_{2}=E_{2}(\delta, f) \subset[0,1)^{l} \times(1,+\infty)^{p-l}$ of asymptotically finite logarithmic measure, such that the inequalities
\end{abstract}

$$
\begin{gathered}
M_{f}(r) \leq \mu_{f}(r) \prod_{i \in I} \frac{1}{\left(1-r_{i}\right)^{1+\delta}} \ln ^{p / 2+\delta}\left(\mu_{f}(r) \prod_{i \in I} \frac{1}{1-r_{i}}\right)\left(\prod_{j \in J} \ln r_{j}\right)^{p+\delta}, \\
M_{f}(r, t) \leq \mu_{f}(r) \prod_{i \in I} \frac{1}{\left(1-r_{i}\right)^{1 / 2+\delta}} \ln ^{p / 4+\delta}\left(\mu_{f}(r) \prod_{i \in I} \frac{1}{1-r_{i}}\right)\left(\prod_{j \in J} \ln r_{j}\right)^{p / 2+\delta} .
\end{gathered}
$$

hold for all $r \in T \backslash E_{1}$ and for all $r \in T \backslash E_{2}$ a.s. in $t$, respectively. Also sharpness of the obtained inequalities is proved.

1. Introduction and the main result. Let $f$ be an analytic function in the disc $\mathbb{D}_{R}=$ $\{z:|z|<R\}, 0<R \leq+\infty$, represented by the power series

$$
f(z)=\sum_{n=0}^{+\infty} a_{n} z^{n} .
$$

Put $\mathbb{D}=\mathbb{D}_{1}, \mathbb{C}=\mathbb{D}_{+\infty}$. For $r \in(0, R)$ we denote

$$
M_{f}(r)=\max \{|f(z)|:|z|=r\}, \mu_{f}(r)=\max \left\{\left|a_{n}\right| r^{n}: n \geq 0\right\} .
$$

It is well known ([1], [2, p. 9], [3, 4], [5, p. 28], [6, 7, 8]) that for each nonconstant entire function $f(z)$ and for every $\varepsilon>0$ there exists a set $E(\varepsilon, f) \subset[1,+\infty)$ such that Wiman's inequality

$$
M_{f}(r) \leq \mu_{f}(r)\left(\ln \mu_{f}(r)\right)^{1 / 2+\varepsilon}
$$

2010 Mathematics Subject Classification: 30B20, 30D20.

Keywords: maximum modulus; maximal term; multiple power series; Wiman's type inequality. doi:10.15330/ms.49.1.29-51 
holds for all $r \in[1,+\infty) \backslash E(\varepsilon, f)$, where the set $E(\varepsilon, f)$ has finite logarithmic measure on $\mathbb{R}_{+}$, i.e.

$$
\int_{E(\varepsilon, f)} \frac{d r}{r}<+\infty .
$$

Also, new descriptions of the magnitude of the exceptional set $E(\varepsilon, f)$ in inequality $(2)$ were received in papers $[6,7]$.

Let $f(z)$ be an analytic function in the unit disc $\mathbb{D}$ of form (1). For a such function $f(z)$ and for every $\delta>0$ there exists a set $E_{f}(\delta) \subset(0,1)$ of finite logarithmic measure on $(0,1)$, i.e.

$$
\int_{E_{f}(\delta)} \frac{d r}{1-r}<+\infty
$$

such that for all $r \in(0,1) \backslash E_{f}(\delta)$ the inequality

$$
M_{f}(r) \leq \frac{\mu_{f}(r)}{(1-r)^{1+\delta}} \ln ^{1 / 2+\delta} \frac{\mu_{f}(r)}{1-r}
$$

holds ([12]). One can find similar inequality for analytic functions in the unit disc in [11].

Also in [12] was noted that for the function $g(z)=\sum_{n=1}^{+\infty} \exp \left\{n^{\varepsilon}\right\} z^{n}, \varepsilon \in(0,1)$ one has

$$
\lim _{r \rightarrow 1-0} \frac{M_{g}(r)}{\frac{\mu_{g}(r)}{1-r} \ln ^{1 / 2} \frac{\mu_{g}(r)}{1-r}} \geq C>0 .
$$

In [13] it is proved that by some conditions inequality (2) holds for every analytic function of form (1) in the unit disc outside an exceptional set.

Some analogues of Wiman's inequality for entire functions of several complex variables can be found in [14]-[23], for analytic functions in the polydisc $\mathbb{D}^{p}, p \geq 2$, in $[24,25,26]$, for analytic functions in the unbounded cylinder $\mathbb{D} \times \mathbb{C}$ in $[27,29]$, respectively.

In [14] there is considered entire functions of $p$ complex variables

$$
f(z)=f\left(z_{1}, \ldots, z_{p}\right)=\sum_{\|n\|=0}^{+\infty} a_{n} z^{n}
$$

where $z^{n}=z_{1}^{n_{1}} \ldots z_{p}^{n_{p}}, p \in \mathbb{N}, n=\left(n_{1}, \ldots, n_{p}\right) \in \mathbb{Z}_{+}^{p},\|n\|=\sum_{j=1}^{p} n_{j}$. For $r=\left(r_{1}, \ldots, r_{p}\right) \in$ $\mathbb{R}_{+}^{p}$ denote

$$
\begin{gathered}
\triangle_{r_{0}}=\left\{t \in \mathbb{R}_{+}^{p}: t_{j} \geq r_{j}^{0}, j \in\{1, \ldots, p\}\right\}, \ln _{2} x=\ln \ln x, r^{\wedge}=\min _{1 \leq i \leq p} r_{i}, \\
M_{f}(r)=\max \left\{|f(z)|:\left|z_{1}\right|=r_{1}, \ldots,\left|z_{p}\right|=r_{p}\right\}, \\
\mu_{f}(r)=\max \left\{\left|a_{n}\right| r_{1}^{n_{1}} \ldots r_{p}^{n_{p}}: n \in \mathbb{Z}_{+}^{p}\right\}, \mathfrak{M}_{f}(r)=\sum_{\|n\|=0}^{+\infty}\left|a_{n}\right| r^{n} .
\end{gathered}
$$

By $\Lambda^{p}$ we denote the class of entire functions of form (3) such that $\frac{\partial}{\partial z_{j}} f(z) \not \equiv 0$ in $\mathbb{C}^{p}$ for any $j \in\{1, \ldots, p\}$. A subset $E$ of $\mathbb{R}_{+}^{p}$ is a set of asymptotically finite logarithmic measure on $\mathbb{R}_{+}^{p}$ if $E$ is Lebesgue measurable in $\mathbb{R}_{+}^{p}$ and there exists an $R \in \mathbb{R}_{+}^{p}$ such that $E \cap \triangle_{r_{0}}$ is a set of finite logarithmic measure, i.e.

$$
\int_{E \cap \triangle_{r_{0}}} \cdots \int \prod_{j=1}^{p} \frac{d r_{j}}{r_{j}}<+\infty .
$$


Theorem A ([14]). Let $f \in \Lambda^{p}$ and $\delta>0$.

a) Then there exist $r_{0} \in \mathbb{R}^{p}$ and a subset $E$ of $\triangle_{r_{0}}$ of finite logarithmic measure such that for $r \in \triangle_{r_{0}} \backslash E$ we have

$$
\mathfrak{M}_{f}(r) \leq \mu_{f}(r)\left(\prod_{i=1}^{p} \ln ^{p-1} r_{i} \cdot \ln ^{p} \mu_{f}(r)\right)^{1 / 2+\delta} .
$$

b) If for some $\alpha \in \mathbb{R}_{+}^{p}: \mathfrak{M}(r) \geq \exp \left(r^{\alpha}\right)=\exp \left(r_{1}^{\alpha_{1}} \ldots r_{p}^{\alpha_{p}}\right)$ as $r^{\wedge} \rightarrow+\infty$ or, more generally, for each $\beta>0$

$$
\int \ldots \int \frac{\prod_{i=1}^{p} d r_{i}}{r_{1} r_{2} \ldots r_{p} \ln ^{\beta} \mathfrak{M}_{f}(r)}<+\infty, \text { as } S^{\wedge} \rightarrow+\infty
$$

then there exist $r_{0} \in \mathbb{R}^{p}$ and a subset $E$ of $\triangle_{r_{0}}$ of finite logarithmic measure such that for $r \in \triangle_{r_{0}} \backslash E$ we have

$$
\mathfrak{M}_{f}(r) \leq \mu_{f}(r) \ln ^{p / 2+\delta} \mu_{f}(r)
$$

In [25] there were proved some analogues of Wiman's inequality for analytic functions $f$ represented by the series of form (3) with the domain of convergence

$$
\mathbb{D}^{p}=\left\{z \in \mathbb{C}^{p}:\left|z_{j}\right|<1, j \in\{1, \ldots, p\}\right\} .
$$

By $\mathcal{A}_{0}^{p}$ we denote the class of such analytic functions that for any $i \in\{1, \ldots, p\}$ we have $r_{i} \frac{\partial}{\partial r_{i}} \ln \mathfrak{M}_{f}(r)+\ln r_{i}>1$ for $r \in\left(t_{0}, 1\right)^{p}$.

Theorem B ([25]). Let $f \in \mathcal{A}_{0}^{p}$. For every $\delta>0$ there exists a set $E=E(f, \delta) \subset[0,1)^{p}$ of asymptotically finite logarithmic measure such that for all $r \in[0,1)^{p} \backslash E$ we have

$$
M_{f}(r) \leq \mu_{f}(r) \prod_{j=1}^{p} \frac{1}{\left(1-r_{j}\right)^{1+\delta}} \cdot \ln ^{p / 2+\delta}\left\{\mu_{f}(r) \prod_{j=1}^{p} \frac{1}{1-r_{j}}\right\} .
$$

In [27] one can find some analogues of Wiman's inequality for analytic functions represented by the series

$$
f(z)=f\left(z_{1}, z_{2}\right)=\sum_{n+m=0}^{+\infty} a_{n m} z_{1}^{n} z_{2}^{m}
$$

with the domain of convergence $\mathbb{D} \times \mathbb{C}=\left\{z \in \mathbb{C}^{2}:\left|z_{1}\right|<1, z_{2} \in \mathbb{C}\right\}$.

By $\mathcal{A}_{1}^{2}$ we denote the class of analytic functions of form (5) with the domain of convergence $\mathbb{D} \times \mathbb{C}$ and

$$
\frac{\partial}{\partial z_{2}} f\left(z_{1}, z_{2}\right) \not \equiv 0 \text { in } \mathbb{D} \times \mathbb{C}, r_{1} \frac{\partial}{\partial r_{1}} \ln \mathfrak{M}_{f}(r)+\ln r_{1}>1\left(\forall\left(r_{1}, r_{2}\right) \in\left(r_{1}^{0}, 1\right) \times\left(r_{2}^{0},+\infty\right)\right) .
$$

We say that $E \subset T_{1}=(0,1) \times \mathbb{R}_{+}$is set of asymptotically finite logarithmic measure on $T_{1}$ if there exists $r_{0} \in T_{1}$ such that

$$
\nu_{\ln }\left(E \cap \triangle_{r_{0}}\right):=\iint_{E \cap \triangle_{r_{0}}} \frac{d r_{1} d r_{2}}{\left(1-r_{1}\right) r_{2}}<+\infty,
$$


i.e. the set $E \cap \triangle_{r_{0}}$ is a set of finite logarithmic measure on $T_{1}$.

Theorem $\mathbf{C}([27])$. Let $f \in \mathcal{A}_{1}^{2}$. For every $\delta>0$ there exists a set $E=E(\delta, f) \subset T_{1}$ of asymptotically finite logarithmic measure such that for all $r \in T_{1} \backslash E$ we obtain

$$
M_{f}(r) \leq \frac{\mu_{f}(r)}{\left(1-r_{1}\right)^{1+\delta}} \ln ^{1+\delta} \frac{\mu_{f}(r)}{1-r_{1}} \cdot \ln ^{1 / 2+\delta} r_{2}
$$

By $\mathcal{A}_{0}^{p}\left(\mathbb{T}_{l}\right), p \geq 1,1 \leq l \leq p$, denote the class of analytic functions of form (3) with the domain of convergence

$\mathbb{T}_{l}=\left\{z=\left(z_{1}, \ldots, z_{p}\right) \in \mathbb{C}^{p}:\left|z_{k}\right|<1, z_{j} \in \mathbb{C}, k \in\{1, \ldots, l\}, j \in\{l+1, \ldots, p\}\right\}=\mathbb{D}^{l} \times \mathbb{C}^{p-l}$,

and by $\mathcal{A}^{p}(\mathbb{T})$ denote a subclass of the functions $f \in \mathcal{A}_{0}^{p}\left(\mathbb{T}_{l}\right)$ such that

$$
\frac{\partial}{\partial z_{j}} f\left(z_{1}, \ldots, z_{p}\right) \not \equiv 0\left(\forall z \in \mathbb{T}_{l} \text { and } \forall j \in\{l+1, \ldots, p\}\right)
$$

and there exists $r_{0} \in \mathbb{R}_{+}^{p}$ such that for any $k \in\{1, \ldots, l\}$

$$
r_{k} \frac{\partial}{\partial r_{k}} \ln \mathfrak{M}_{f}(r)+\ln r_{k}>1\left(\forall r \in\left(r_{1}^{0}, 1\right)^{l} \times\left(r_{2}^{0},+\infty\right)^{p-l}\right) .
$$

The aim of this paper is to prove some analogues of Wiman's inequality for analytic functions $f$ represented by the series of the form (3) with the domain of convergence $\mathbb{T}:=$ $\mathbb{T}_{l}=\mathbb{D}^{l} \times \mathbb{C}^{p-l}, l \in \mathbb{N}, 1 \leq l<p$. A natural problem arises: to prove sharp analogues of the Wiman type inequalities for analytic functions in $\mathbb{T}$.

2. Wiman's type inequality for analytic functions in $\mathbb{T}_{l}$. For $r=\left(r_{1}, \ldots, r_{p}\right) \in$ $T:=[0,1)^{l} \times[0,+\infty)^{p-l}$ and a function $f \in \mathcal{A}^{p}\left(\mathbb{T}_{l}\right)$ we denote

$$
\begin{gathered}
\triangle_{r}=\left\{\left(t_{1}, \ldots, t_{p}\right) \in T: t_{j}>r_{j}, j \in\{1, \ldots, p\}\right\}, I=\{1, \ldots, l\}, J=\{l+1, \ldots, p\}, \\
M_{f}(r)=\max \left\{|f(z)|:\left|z_{j}\right| \leq r_{j}, j \in\{1, \ldots, p\}\right\}, \\
\mu_{f}(r)=\max \left\{\left|a_{n}\right| r^{n}:\left(n_{1}, \ldots, n_{p}\right) \in \mathbb{Z}_{+}^{p}\right\}, \mathfrak{M}_{f}(r)=\sum_{\|n\|=0}^{+\infty}\left|a_{n}\right| r^{n} .
\end{gathered}
$$

We say that $E \subset T$ is set of asymptotically finite logarithmic measure on $T$ if there exists $r_{0} \in T$ such that

$$
\nu_{\ln }\left(E \cap \triangle_{r_{0}}\right):=\int_{E \cap \triangle_{r_{0}}} \ldots \int \prod_{i \in I} \frac{d r_{i}}{1-r_{i}} \prod_{j \in J} \frac{d r_{j}}{r_{j}}<+\infty .
$$

The set of all such sets is denoted by $\Upsilon$.

Theorem 1. Let $f \in \mathcal{A}^{p}(\mathbb{T})$. For every $\delta>0$ there exists a set $E=E(\delta, f) \subset T, E \in \Upsilon$ such that for all $r \in T \backslash E$ we obtain

$$
M_{f}(r) \leq \mu_{f}(r) \prod_{i \in I} \frac{1}{\left(1-r_{i}\right)^{1+\delta}} \ln ^{p / 2+\delta}\left(\mu_{f}(r) \prod_{i \in I} \frac{1}{1-r_{i}}\right)\left(\prod_{j \in J} \ln r_{j}\right)^{p+\delta} .
$$

In order to prove Theorem 1 we need the following auxiliary results. 
Lemma 1. Let $F$ be a function of the form

$$
F(\sigma)=\int_{\mathbb{R}_{+}^{p}} a(x) e^{\langle\sigma, x\rangle} \nu(d x)
$$

where $a(x): \mathbb{R}_{+}^{p} \rightarrow \mathbb{R}_{+}$is a $\nu$-measurable function, and $\nu$ is a countable-additive measure on $\mathbb{R}_{+}^{p}$ with unbounded support. We assume that for some fixed $\sigma \in \mathbb{R}_{+}^{p}$ there exist

$$
\frac{\partial}{\partial \sigma_{j}} F(\sigma)=\int_{\mathbb{R}_{+}^{p}} x_{j} a(x) e^{\langle\sigma, x\rangle} \nu(d x), \frac{\partial^{2}}{\partial \sigma_{j}^{2}} F(\sigma)=\int_{\mathbb{R}_{+}^{p}} x_{j}^{2} a(x) e^{\langle\sigma, x\rangle} \nu(d x) .
$$

Then

$$
F(\sigma) \leq \frac{c}{c-1} \int_{X_{0}(\sigma)} a(x) e^{\langle\sigma, x\rangle} \nu(d x) .
$$

where $c=c(\sigma)>1$ is arbitrary,

$$
\begin{gathered}
a_{j}=\frac{\partial}{\partial \sigma_{j}} \ln F(\sigma), b_{j}=\frac{\partial^{2}}{\partial \sigma_{j}^{2}} \ln F(\sigma), \\
X_{0}(\sigma)=\left\{x=\left(x_{1}, \ldots, x_{p}\right) \in \mathbb{R}_{+}^{p}:\left|x_{j}-a_{j}\right| \leq \sqrt{c p b_{j}}, 1 \leq j \leq p\right\} .
\end{gathered}
$$

Proof of Lemma 1. We repeat the arguments from the proof of Lemma 1 from [20]. Consider random variables $\xi_{j}=x_{j}, 1 \leq j \leq p$ on the probability space $\mathbb{R}_{+}^{p}$ with the following measure

$$
P_{\sigma}(d x)=P(d x)=\frac{a(x)}{F(\sigma)} e^{\langle\sigma, x\rangle} \nu(d x) .
$$

So, the expectation of the variable $\xi_{j}$

$$
\mathbf{E} \xi_{j}=\int_{\mathbb{R}_{+}^{p}} x_{j} a(x) e^{\langle\sigma, x\rangle} P(d x)=\frac{1}{F(\sigma)} \frac{\partial F(\sigma)}{\partial \sigma_{j}}=a_{j},
$$

and the variance of $\xi_{j}$

$$
\mathbf{D} \xi_{j}=M \xi_{j}^{2}-\left(M \xi_{j}\right)^{2}=\frac{1}{F(\sigma)} \frac{\partial^{2} F(\sigma)}{\partial \sigma_{j}^{2}}-\left(\frac{1}{F(\sigma)} \frac{\partial F(\sigma)}{\partial \sigma_{j}}\right)^{2}=\frac{\partial}{\partial \sigma_{j}}\left(\frac{1}{F(\sigma)} \frac{\partial F(\sigma)}{\partial \sigma_{j}}\right)=b_{j} .
$$

We put $d_{j}=\sqrt{p c b_{j}}, A_{j}=\left\{x \in \mathbb{R}_{+}^{p}:\left|\xi_{j}-\mathbf{E} \xi_{j}\right| \geq d_{j}\right\}, A=\bigcup_{j=1}^{p} A_{j}$. We have $X_{0}(\sigma)=\bar{A}$. Using $p$ times the Bienayme-Chebyshev inequality (see $[35,36]) P\left(A_{j}\right)=P\left\{x: \mid \xi_{j}(x)-\right.$ $\left.\mathbf{E} \xi_{j} \mid \geq d_{j}\right\} \leq \mathbf{D} \xi_{j} / d_{j}^{2}$ we obtain

$$
P(A) \leq \sum_{j=1}^{p} P\left(A_{j}\right) \leq \sum_{j=1}^{p} \frac{1}{d_{j}^{2}} D \xi_{j}=\frac{1}{c}
$$

Therefore,

$$
F(\sigma)=\int_{X_{0}(\sigma)} f(x) e^{\langle\sigma, x\rangle} \nu(d x)+F(\sigma) \int_{A} \frac{f(x)}{F(\sigma)} e^{\langle\sigma, x\rangle} \nu(d x)=
$$




$$
=\int_{X_{0}(\sigma)} f(x) e^{\langle\sigma, x\rangle} \nu(d x)+F(\sigma) P(A) \leq \int_{X_{0}(\sigma)} f(x) e^{\langle\sigma, x\rangle} \nu(d x)+\frac{F(\sigma)}{c} .
$$

Hence, we have inequality (8).

Lemma 2. Let $f \in \mathcal{A}^{p}(\mathbb{T})$ be a function of form (3),

$$
\begin{gathered}
a_{j}=r_{j} \frac{\partial}{\partial r_{j}} \ln \mathfrak{M}_{f}(r), b_{j}=r_{j} \frac{\partial}{\partial r_{j}}\left(r_{j} \frac{\partial}{\partial r_{j}} \ln \mathfrak{M}_{f}(r)\right), C=C(r)>1, \\
X_{0}(r)=\left\{x \in \mathbb{R}_{+}^{p}:\left|x_{j}-a_{j}\right| \leq \sqrt{c p b_{j}}, 1 \leq j \leq p\right\} .
\end{gathered}
$$

Then for all $r \in T$ we have

$$
\mathfrak{M}_{f}(r) \leq \frac{C}{C-1} \sum_{n \in X_{0}(r)}\left|a_{n}\right| r^{n}
$$

Proof. For $\sigma=\left(\sigma_{1}, \ldots, \sigma_{p}\right), r=\left(r_{1}, \ldots, r_{p}\right), r_{j}=e^{\sigma_{j}}$ we have

$$
\mathfrak{M}_{f}(r)=\sum_{\|n\|=0}^{+\infty}\left|a_{n}\right| r^{n}=\int_{\mathbb{R}_{+}^{p}} a(x) e^{\langle\sigma, x\rangle} \nu(d x)=F(\sigma)
$$

where $a(x)=\left|a_{n}\right|$ for $x=n \in \mathbb{Z}_{+}^{p}$ and $\nu$ is the measure such that

$$
\nu(E)=\sum_{n \in E} \delta_{n}(E), \quad \delta_{n}(E)= \begin{cases}1, & n \in E, \\ 0, & n \notin E,\end{cases}
$$

for any bounded set $E \subset \mathbb{R}_{+}^{p}$.

Remark that

$$
\frac{\partial \ln F(\sigma)}{\partial \sigma_{j}}=r_{j} \frac{\partial \ln \mathfrak{M}_{f}(r)}{\partial r_{j}} .
$$

Thus, using Lemma 1 with $c=C, r=e^{\sigma}$ and $X_{0}^{*}(r)=X_{0}(\ln \sigma)=$ we get

$$
\mathfrak{M}_{f}(r)=F(\sigma) \leq \frac{c}{c-1} \int_{X_{0}(\sigma)} a(x) e^{\langle\sigma, x\rangle} \nu(d x)=\frac{C}{C-1} \sum_{n \in X_{0}^{*}(r)}\left|a_{n}\right| r^{n} .
$$

The proof of Lemma 2 is complete.

Lemma 3. Let $\delta>0$. If $f \in \mathcal{A}^{p}(\mathbb{T})$ then there exists a set $E \subset T(E \in \Upsilon)$, such that for all $r \in T \backslash E$ the inequalities

$$
\begin{gathered}
\frac{\partial}{\partial r_{m}} \ln \mathfrak{M}_{f}(r) \leq \frac{1}{1-r_{m}} \prod_{i \in I, i \neq m} \frac{1}{\left(1-r_{i}\right)^{\delta}}\left(\ln \mathfrak{M}_{f}(r) \prod_{j \in J} \ln r_{j}\right)^{1+\delta}, m \in I ; \\
\frac{\partial}{\partial r_{k}} \ln \mathfrak{M}_{f}(r) \leq \prod_{i \in I} \frac{1}{\left(1-r_{i}\right)^{\delta}}\left(\ln \mathfrak{M}_{f}(r) \prod_{j \in J, j \neq k} \ln r_{j}\right)^{1+\delta}, k \in J
\end{gathered}
$$

hold. 
Proof of Lemma 3. Without loss generality we prove inequality (9) for $i=1$. Suppose that $E_{1}^{*} \subset T$ is the set for which inequality (9) with $i=1$ does not hold. Then we choose $r^{0} \in T$ such that $\ln \mathfrak{M}_{f}\left(r^{0}\right)>1$ and $r_{j}^{0}>e, j \in J, r_{i}^{0}>1 / 2, i \in I$

$$
\begin{gathered}
\nu_{\ln }\left(E_{1}^{*} \cap \triangle_{r^{0}}\right) \leq \int_{E_{1}^{*} \cap \triangle_{r^{0}}} \ldots \int \prod_{i \in I} \frac{1}{1-r_{i}} \prod_{j \in J} \frac{1}{r_{j}} d r_{1} \ldots d r_{p} \leq \\
\leq \int_{E_{1}^{*} \cap \triangle_{r^{0}}} \ldots \int \frac{\left(1-r_{1}\right) \frac{\partial}{\partial r_{1}} \ln \mathfrak{M}_{f}(r) \prod_{i=2 m}^{l}\left(1-r_{i}\right)^{\delta} d r_{1} \ldots d r_{p}}{\prod_{i \in I}\left(1-r_{i}\right) \prod_{j \in J} r_{j}\left(\ln \mathfrak{M}_{f}(r)\right)^{1+\delta}\left(\prod_{j \in J} \ln r_{j}\right)^{1+\delta}} .
\end{gathered}
$$

Consider the mapping $V: T \rightarrow \mathbb{R}_{+}^{p}$, where $V=\left(v_{1}(r), \ldots, v_{p}(r)\right), v_{1}(r)=\ln \mathfrak{M}_{f}(r)$, $v_{2}(r)=r_{2}, \ldots, v_{l-1}(r)=r_{l-1}, v_{l}(r)=\ln r_{l}, \ldots, v_{p}(r)=\ln r_{p}$.

So

$$
J_{2}:=\frac{D\left(v_{1}(r), \ldots, v_{p}(r)\right)}{D\left(r_{1}, \ldots, r_{p}\right)}=\left|\begin{array}{ccc}
\frac{\partial v_{1}}{\partial r_{1}} & \cdots & \frac{\partial v_{1}}{\partial r_{p}} \\
\vdots & \ddots & \vdots \\
\frac{\partial v_{p}}{\partial r_{1}} & \cdots & \frac{\partial v_{p}}{\partial r_{2} p}
\end{array}\right|=\prod_{j \in J} \frac{1}{r_{j}} \frac{\partial}{\partial r_{1}} \ln \mathfrak{M}_{f}(r) .
$$

Therefore

$$
\nu_{\ln }\left(E_{1}^{*} \cap \triangle_{r^{0}}\right) \leq \int_{\cup\left(E_{2}^{*} \cap \triangle_{r^{0}}\right)} \ldots \int_{1} \frac{d v_{1} \ldots d v_{p}}{v_{1}^{1+\delta}\left(1-v_{2}\right)^{1-\delta} \ldots\left(1-v_{l-1}\right)^{1-\delta} \cdot v_{l}^{1+\delta} \ldots v_{p}^{1+\delta}}<+\infty .
$$

Without loss generality we prove inequality (9) for $j=p$. Let $E_{p} \subset T$ be a set for which inequality (9) does not hold with $j=p$

$$
\nu_{\ln }\left(E_{1}^{*} \cap \triangle_{r^{0}}\right) \leq \int_{E_{p}^{*} \cap \triangle_{r^{0}}} \ldots \int \frac{r_{p} \frac{\partial}{\partial r_{p}} \ln \mathfrak{M}_{f}(r) \prod_{i \in I}\left(1-r_{i}\right)^{\delta} d r_{1} \ldots d r_{p}}{\left(\ln \mathfrak{M}_{f}(r) \prod_{j=l+1}^{p-1} \ln r_{j}\right)^{1+\delta} \prod_{i \in I}\left(1-r_{i}\right) \prod_{j \in J} r_{j}} .
$$

Let $r^{0}$ be such that $\ln \mathfrak{M}_{f}\left(r^{0}\right)>1$. Define the mapping $W: T \rightarrow T$, where $W=$ $\left(w_{1}(r), \ldots, w_{p}(r)\right)$, and

$$
w_{1}(r)=r_{1}, \ldots, w_{l}(r)=r_{l}, \ldots, w_{l+1}(r)=r_{l+1}, \ldots, v_{p-1}(r)=\ln r_{p-1}, \ldots, w_{p}(r)=\ln \mathfrak{M}_{f}(r) .
$$

So,

$$
J_{3}:=\left|\begin{array}{ccc}
\frac{\partial w_{1}}{\partial r_{1}} & \cdots & \frac{\partial w_{1}}{\partial r_{p}} \\
\vdots & \ddots & \vdots \\
\frac{\partial w_{p}}{\partial r_{1}} & \cdots & \frac{\partial w_{p}}{\partial r_{2} p}
\end{array}\right|=\prod_{j=l+1}^{p-1} \frac{1}{r_{j}} \cdot \frac{\partial}{\partial r_{p}} \ln \mathfrak{M}_{f}(r)
$$

Therefore

$$
\begin{aligned}
\nu_{\ln }\left(E_{1}^{*} \cap \triangle_{r^{0}}\right) & \leq \int_{E_{p}^{*} \cap \triangle_{r^{0}}} \ldots \int \frac{\frac{\partial}{\partial r_{p}} \ln \mathfrak{M}_{f}(r) \prod_{i \in I}\left(1-r_{i}\right)^{\delta} d r_{1} \ldots d r_{p}}{\left(\ln \mathfrak{M}_{f}(r) \prod_{j=l+1}^{p-1} \ln r_{j}\right)^{1+\delta} \prod_{i \in I}\left(1-r_{i}\right) \prod_{j \in J} r_{j}} \leq \\
& \leq \int_{E_{p}^{*} \cap \triangle_{r^{0}}} \ldots \int \frac{d w_{1} \ldots d w_{p}}{\prod_{i \in I}\left(1-w_{i}\right)^{1-\delta} \prod_{j \in J} w_{j}^{1+\delta}}<+\infty .
\end{aligned}
$$

It remains to remark, that if $E_{j} \in \Upsilon, j \in\{1, \ldots, p\}$ then $\bigcup_{j=0}^{p} E_{j} \in \Upsilon$. 
Proof of Theorem 1. Let $E^{\prime}$ and $E_{0}$ be the exceptional sets from Theorem 1 and Lemma 1, respectively. Then for $E=E^{\prime} \cup E_{0}$ and $\delta>0$ we get for all $r \in T \backslash E$

$$
\begin{gathered}
\mathfrak{M}_{f}(r) \leq \frac{C}{C+1} \mu_{f}(r) \prod_{j=1}^{p}\left(2 \sqrt{c p b_{j}}+2\right) \leq C^{*} \mu_{f}(r) \prod_{j=1}^{p} \sqrt{\frac{\partial^{2}}{\partial^{2} r_{j}} \ln \mathfrak{M}_{f}(r) \leq} \\
\leq C^{*} \mu_{f}(r) \prod_{m \in B}\left[\frac{1}{1-r_{m}} \prod_{i \in I, i \neq m} \frac{1}{\left(1-r_{i}\right)^{\delta}}\left(\frac{\partial}{\partial r_{m}} \ln \mathfrak{M}_{f}(r) \prod_{j \in J} \ln r_{j}\right)^{1+\delta}\right]^{1 / 2} \times \\
\times \prod_{k \in D}\left[\prod_{i \in I} \frac{1}{\left(1-r_{i}\right)^{\delta}}\left(\frac{\partial}{\partial r_{k}} \ln \mathfrak{M}_{f}(r) \prod_{j \in J, j \neq k} \ln r_{j}\right)^{1+\delta}\right]^{1 / 2} \leq \\
\leq C^{*} \mu_{f}(r) \prod_{m \in B}\left[\frac { 1 } { 1 - r _ { m } } \prod _ { i \in I , i \neq m } \frac { 1 } { ( 1 - r _ { i } ) ^ { \delta } } \left(\left(\frac{1}{1-r_{m}} \prod_{i \in I, i \neq m} \frac{1}{\left(1-r_{i}\right)^{\delta}} \times\right.\right.\right. \\
\left.\left.\left.\times\left(\ln \mathfrak{M}_{f}(r) \prod_{j \in J} \ln r_{j}\right)^{1+\delta}\right) \prod_{j \in J} \ln r_{j}\right)^{1+\delta}\right]^{1 / 2} \times \prod_{j \in D}\left[\prod_{i \in I} \frac{1}{\left(1-r_{i}\right)^{\delta}}\left(\left(\prod_{i \in I} \frac{1}{\left(1-r_{i}\right)^{\delta}}\left(\ln \mathfrak{M}_{f}(r) \prod_{j \in J, j \neq k} \ln r_{j}\right)^{1+\delta}\right) \prod_{j \in J, j \neq k} \ln r_{j}\right)^{1+\delta}\right]^{1 / 2} \leq \\
\leq \mu_{f}(r) \prod_{i \in I} \frac{1}{\left(1-r_{i}\right)^{1+2 p^{2} \delta}} \ln ^{p / 2+2 p^{2} \delta} \mathfrak{M}_{f}(r) \prod_{j \in J}\left(\ln r_{j}\right)^{p+2 p^{2} \delta} .
\end{gathered}
$$

Then

$$
\begin{gathered}
\ln \mathfrak{M}_{f}(r) \leq \ln \mu_{f}(r)+ \\
+\left(1+2 p^{2} \delta\right) \sum_{i \in I} \ln \frac{1}{1-r_{i}}+\left(\frac{p}{2}+2 p^{2} \delta\right) \ln \ln \mathfrak{M}_{f}(r)+\left(p+2 p^{2} \delta\right) \sum_{j \in J} \ln \ln r_{j}, \\
\ln \mathfrak{M}_{f}(r)-\left(p / 2+2 p^{2} \delta\right) \ln \ln \mathfrak{M}_{f}(r) \leq\left(p+2 p^{2} \delta\right) \ln \left(\mu_{f}(r) \prod_{i \in I} \frac{1}{\left(1-r_{i}\right)} \prod_{j \in J} \ln r_{j}\right), \\
\mathfrak{M}_{f}(r) \leq \prod_{i \in I} \frac{1}{\left(1-r_{i}\right)^{1+2 p^{2} \delta}} \ln ^{p / 2+2 p^{2} \delta}\left(\mu_{f}(r) \prod_{i \in I} \frac{1}{\left(1-r_{i}\right)} \prod_{j \in J} \ln r_{j}\right) \prod_{j \in J}\left(\ln r_{j}\right)^{p+2 p^{2} \delta} \leq \\
\leq \mu_{f}(r) \prod_{i \in I} \frac{1}{\left(1-r_{i}\right)^{1+\delta_{1}}} \ln ^{p / 2+\delta_{1}}\left(\mu_{f}(r) \prod_{i \in I} \frac{1}{\left(1-r_{i}\right)}\right)\left(\sum_{j \in J} \ln \ln r_{j}\right)^{p / 2+\delta_{1}} \prod_{j \in J}\left(\ln r_{j}\right)^{p+2 \delta_{1}}= \\
=\mu_{f}(r) \prod_{i \in I} \frac{1}{\left(1-r_{i}\right)^{1+\delta_{1}}} \ln ^{p / 2+\delta_{1}}\left(\mu_{f}(r) \prod_{i \in I} \frac{1}{\left(1-r_{i}\right)}\right) \prod_{j \in J}\left(\ln r_{j}\right)^{p / 2+\delta_{1}} \ln \left[\prod_{j \in J}\left(\ln r_{j}\right)^{p+2 \delta_{1}}\right] \leq \\
\leq \mu_{f}(r) \prod_{i \in I} \frac{1}{\left(1-r_{i}\right)^{1+\delta_{1}}} \ln ^{p / 2+\delta_{1}}\left(\mu_{f}(r) \prod_{i \in I} \frac{1}{1-r_{i}}\right)\left(\prod_{j \in J} \ln r_{j}\right)^{p+2 \delta_{1}} .
\end{gathered}
$$

3. Sharpness of Theorem 1. Remark that the exponent $1+\delta$ at $\prod_{i \in I} \frac{1}{1-r_{i}}$ in inequality (6) cannot be replaced a number smaller than 1 . Suppose that one can replace this exponent 
by $\sigma \in(0,1)$. Consider the function

$$
f(z)=f\left(z_{1}, \ldots, z_{p}\right)=\prod_{i \in I} \frac{1}{1-r_{i}} \prod_{j \in J} \gamma_{j}\left(z_{j}\right),
$$

where $\gamma_{j}\left(z_{j}\right)$ are entire functions such that $\ln \mu_{\gamma_{j}}\left(r_{j}\right)<r_{j}, r \rightarrow+\infty, j \in J$. Then $M_{f}(r)=$ $\prod_{i \in I} \frac{1}{1-r_{i}} \prod_{j \in J} M_{\gamma_{j}}\left(z_{j}\right)$ and $\mu_{f}(r)=\prod_{j \in D} \mu_{\gamma_{j}}\left(r_{j}\right)$. Denote $r^{\prime}(\varepsilon, p)$ such that for all $r_{j}>$ $r^{\prime}(\varepsilon, p), i \in I$ we have

$$
\left(\frac{1}{\left(1-r_{i}\right)^{1-\varepsilon}}\right)^{2 / p}-\ln \frac{1}{1-r_{i}}>e, i \in I
$$

and there exists $r_{0} \in T$ such that

$$
\begin{aligned}
& \triangle_{r_{0}} \cap B^{\prime}=\left\{r \in\left[r^{\prime}(\varepsilon) ; 1\right)^{l} \times[e ;+\infty)^{p-l},(\forall i \in I, \forall j \in J):\left(\frac{1}{\left(1-r_{i}\right)^{1-\varepsilon}}\right)^{2 / p}-\right. \\
& \left.-\ln ^{2} \frac{1}{1-r_{i}}>r_{j}^{2}\right\} \cap \triangle_{r_{0}} \subset\left\{r \in\left[r^{\prime}(\varepsilon) ; 1\right)^{l} \times[e ;+\infty)^{p-l},(\forall i \in I, \forall j \in J):\right. \\
& \left.\left(\frac{1}{\left(1-r_{i}\right)^{1-\varepsilon}}\right)^{2 / p}-\ln \frac{1}{1-r_{i}}\left(\ln r_{j}\right)^{\frac{p-l}{p}}>r_{j}\left(\ln r_{j}\right)^{\frac{p-l}{p}}\right\} \cap \triangle_{r_{0}} \subset \\
& \subset\left\{r \in\left[r^{\prime}(\varepsilon) ; 1\right)^{l} \times[e ;+\infty)^{p-l},(\forall i \in I, \forall j \in J): \frac{1}{1-r_{i}}>\right. \\
& \left.>\frac{1}{\left(1-r_{i}\right)^{\varepsilon}}\left(\ln \frac{1}{1-r_{i}}+r_{j}\right)^{p / 2}\left(\ln r_{j}\right)^{p}\right\} \cap \triangle_{r_{0}} \subset \\
& \subset\left\{r \in\left[r^{\prime}(\varepsilon) ; 1\right)^{l} \times[e ;+\infty)^{p-l},(\forall i \in I, \forall j \in J): \prod_{i \in I} \frac{1}{1-r_{i}} \prod_{j \in J} \mu_{\gamma_{j}}\left(r_{j}\right)>\right. \\
& \left.>\prod_{i \in I} \frac{1}{\left(1-r_{i}\right)^{\varepsilon}} \prod_{j \in J} \mu_{\gamma_{j}}\left(r_{j}\right) \ln ^{p / 2}\left(\prod_{i \in I} \frac{1}{1-r_{i}} \prod_{j \in J} \mu_{\gamma_{j}}\left(r_{j}\right)\right) \prod_{j \in J}\left(\ln r_{j}\right)^{p}\right\} \cap \triangle_{r_{0}} \subset \\
& \subset\left\{r \in\left[r^{\prime}(\varepsilon) ; 1\right)^{l} \times[e ;+\infty)^{p-l}:(\forall i \in I, \forall j \in J) M_{f}(r)>\right. \\
& \left.>\mu_{f}(r) \prod_{i \in I} \frac{1}{\left(1-r_{i}\right)^{\varepsilon}} \ln ^{p / 2}\left(\mu_{f}(r) \prod_{i \in I} \frac{1}{1-r_{i}}\right) \prod_{j \in J}\left(\ln r_{j}\right)^{p}\right\} \cap \triangle_{r_{0}}=B \cap \triangle_{r_{0}} \text {. }
\end{aligned}
$$

Then the measure of the set $B$

$$
\begin{aligned}
& \nu_{\ln }(B)=\int \ldots \int \prod_{B \cap \triangle_{r_{0}}} \frac{d r_{i}}{1-r_{i}} \prod_{j \in J} \frac{d r_{j}}{r_{j}} \geq \int_{B^{\prime} \cap \triangle_{r_{0}}} \ldots \int \prod_{i \in I} \frac{d r_{i}}{1-r_{i}} \prod_{j \in J} \frac{d r_{j}}{r_{j}} \geq \\
& \geq \int_{r^{\prime}(\varepsilon, p)}^{1} \ldots \int_{r^{\prime}(\varepsilon, p)}^{1}\left(\int_{1}^{\sqrt{\left(\frac{1}{\left(1-r_{1}\right)^{1-\varepsilon}}\right)^{2 / p}-\ln ^{2} \frac{1}{1-r_{1}}}} \ldots \int_{1}^{\sqrt{\left(\frac{1}{\left(1-r_{1}\right)^{1-\varepsilon}}\right)^{2 / p}-\ln ^{2} \frac{1}{1-r_{1}}}} \prod_{j \in J} \frac{d r_{j}}{r_{j}}\right) \prod_{i \in I} \frac{d r_{i}}{1-r_{i}}= \\
& =\frac{p-l}{2} \int_{r^{\prime}(\varepsilon, p)}^{1} \ldots \int_{r^{\prime}(\varepsilon, p)}^{1} \ln \left(\left(\frac{1}{1-r_{1}}\right)^{2 / p}-\ln \frac{1}{1-r_{1}}\right) \prod_{i \in I} \frac{d r_{i}}{1-r_{i}} \geq \\
& \geq \frac{p-l}{2} \int_{r^{\prime}(\varepsilon, p)}^{1} \ldots \int_{r^{\prime}(\varepsilon, p)}^{1} \prod_{i \in I} \frac{d r_{i}}{1-r_{i}}=+\infty
\end{aligned}
$$


We remark that any of the exponents 1 and $p / 2$ in the inequality of Theorem 4 cannot be replaced by a smaller number. It follows from such a statement.

Theorem 2. There exist a function $f \in \mathcal{A}^{p}\left(\mathbb{T}_{l}\right)$ and a constant $C>0$ such that

$$
E=\left\{r \in T: M_{f}(r)>C \mu_{f}(r) \prod_{i \in I} \frac{1}{1-r_{i}} \ln ^{p / 2}\left(\mu_{f}(r) \prod_{i \in I} \frac{1}{1-r_{i}}\right)\right\}
$$

has asymptotically infinite logarithmic measure.

Proof of Theorem 2. Let us consider the function

$$
f(z)=f\left(z_{1}, \ldots, z_{p}\right)=\prod_{i \in D} \sum_{n_{i}=0}^{+\infty} \frac{z_{i}^{n_{i}}}{n_{i} !} \prod_{i \in I} \sum_{n_{j}=0}^{+\infty} e^{n_{j}^{\varepsilon}} z_{j}^{n_{j}}=\prod_{i \in I} \psi\left(z_{i}\right) \prod_{j \in J} \varphi\left(z_{j}\right), \varepsilon \in(0,1) .
$$

Remark, that for this function there exists $r_{0}$ such that

$$
\begin{gathered}
\triangle_{r_{0}} \cap\left\{r \in T: M_{f}(r)>\mu_{f}(r) \prod_{i \in I} \frac{1}{\left(1-r_{i}\right)^{1+\delta}} \ln ^{p / 2+\delta}\left\{\mu_{f}(r) \prod_{i \in I} \frac{1}{1-r_{i}} \prod_{j \in J} \ln ^{p} r_{j}\right\} \subset\right. \\
\subset \triangle_{r_{0}} \cap\left\{r \in T: M_{f}(r)>\mu_{f}(r) \prod_{i \in I} \frac{1}{\left(1-r_{i}\right)^{1+\delta}} \ln ^{p / 2+2 \delta}\left\{\mu_{f}(r) \prod_{i \in I} \frac{1}{1-r_{i}}\right\}\right\} .
\end{gathered}
$$

Then

$$
\begin{gathered}
M_{\varphi}\left(r_{i}\right) \geq C_{1}(\varepsilon) \frac{\mu_{\varphi}\left(r_{i}\right)}{1-r_{i}} \ln ^{1 / 2} \frac{\mu_{\varphi}\left(r_{i}\right)}{1-r_{i}}, r_{i}>r_{i}^{0}, i \in I, \\
M_{\psi}\left(r_{j}\right) \geq(\sqrt{2 \pi}-\delta) \mu_{\psi}\left(r_{j}\right) \ln ^{1 / 2} \mu_{\psi}\left(r_{j}\right), \delta>0, r_{j}>r_{j}^{0}, j \in J .
\end{gathered}
$$

So, $M_{f}\left(r_{1}, r_{2}\right)=\prod_{i \in I} M_{\varphi}\left(r_{i}\right) \prod_{j \in J} M_{\psi}\left(r_{j}\right)$ and for $r \in\left(r_{1}^{0}, 1\right)^{l} \times\left(r_{2}^{0},+\infty\right)^{p-l}$ we have

$$
M_{f}(r) \geq(\sqrt{2 \pi}-\delta)^{p-l} C_{1}^{l}(\varepsilon) \mu_{f}(r) \prod_{i \in I} \frac{1}{1-r_{i}}\left(\prod_{i \in I} \ln \frac{\mu_{\varphi}\left(r_{i}\right)}{1-r_{i}} \prod_{j \in J} \ln \mu_{\psi}\left(r_{j}\right)\right)^{1 / 2} .
$$

Consider positive increasing functions $g_{1}(t)=\ln \frac{\mu_{\varphi}(t)}{1-t}$ and $g_{2}(t)=\ln \mu_{\psi}(t)$. We define

$$
\begin{gathered}
A=\left\{r \in T: g_{1}\left(r_{1}\right) \ldots g_{1}\left(r_{l}\right) \cdot g_{2}\left(r_{l+1}\right) \ldots g_{2}\left(r_{p}\right)>\right. \\
\left.>\frac{1}{2^{l}(2 p-l)^{p}}\left(g_{1}\left(r_{1}\right)+\ldots+g_{1}\left(r_{l}\right)+g_{2}\left(r_{l+1}\right)+\ldots+g_{2}\left(r_{p}\right)\right)^{p}\right\} \supset \\
\supset\left\{r \in T:(\forall i \in I)(\forall j \in J) \frac{1}{2}<\frac{g_{1}\left(r_{i}\right)}{g_{2}\left(r_{j}\right)}<2\right\}=E^{*} .
\end{gathered}
$$

Indeed, if $r \in A$ then

$$
\begin{gathered}
g_{1}\left(r_{1}\right) \cdots g_{1}\left(r_{l}\right) g_{2}\left(r_{l+1}\right) \cdots g\left(r_{p}\right) \geq \frac{g_{2}^{2}\left(r_{p}\right)}{2^{l}}=\frac{1}{2^{l}(l+2(p-l))^{p}} \times \\
\times\left(g_{1}\left(r_{1}\right)+\ldots+g_{1}\left(r_{l}\right)+g_{2}\left(r_{l+1}\right)++\ldots+g_{2}\left(r_{p}\right)\right)^{p} \geq \\
\geq \frac{1}{2^{l}(2 p-l)^{2}}\left(g_{1}\left(r_{1}\right)+\ldots+g_{1}\left(r_{l}\right)+g_{2}\left(r_{l+1}\right)+\ldots+g_{2}\left(r_{p}\right)\right)^{p} .
\end{gathered}
$$


There exists the inverse function $g_{2}^{-1}: \mathbb{R}_{+} \rightarrow\left(r_{0}, 1\right)$, which is also increasing.

For $r_{1} \in\left(r_{1}^{0}, 1\right)$ we define $r_{1}^{*}$ and $r_{2}^{*}$ such that

$$
r_{1}^{*}=g_{2}^{-1}\left(\frac{g_{1}\left(r_{1}\right)}{2}\right), r_{2}^{*}=g_{2}^{-1}\left(2 g_{1}\left(r_{1}\right)\right) .
$$

In [27] it was proved that

$$
r<g_{2}^{-1}(r)<\frac{3}{2} r, r \rightarrow+\infty .
$$

Then from inequality (12) for all $r \in E^{*}$ we deduce

$$
\begin{aligned}
M_{f}(r) \geq & \frac{(\sqrt{2 \pi}-\delta)^{p-l} C_{1}^{l}(\varepsilon)}{2^{l}(2 p-l)^{2}} \mu_{f}(r) \prod_{i \in I} \frac{1}{1-r_{i}}\left(\sum_{i \in I} \ln \frac{\mu_{\varphi}\left(r_{i}\right)}{1-r_{i}}+\sum_{j \in J} \ln \mu_{\psi}\left(r_{j}\right)\right)^{p / 2}= \\
& =\frac{(\sqrt{2 \pi}-\delta)^{p-l} C_{1}^{l}(\varepsilon)}{2^{l}(2 p-l)^{2}} \mu_{f}(r) \prod_{i \in I} \frac{1}{1-r_{i}} \ln ^{p / 2}\left\{\mu_{f}(r) \prod_{i \in I} \frac{1}{1-r_{i}}\right\} .
\end{aligned}
$$

It remains to prove that the set $E^{*}$ is a set of infinite asymptotically logarithmic measure. Using (13) we get

$$
\begin{aligned}
& \nu_{\ln }\left(E^{*}\right)=\int_{E^{*} \cap \Delta r_{1}^{0}} \ldots \int_{i \in I} \frac{d r_{i}}{1-r_{i}} \prod_{j \in J} \frac{d r_{j}}{r_{j}}=\int_{r_{0}^{1}}^{1} \ldots \int_{r_{0}^{1}}^{1} \prod_{i \in I} \frac{d r_{i}}{1-r_{i}} \int_{r_{1}^{*}}^{r_{2}^{*}} \prod_{j \in J} \frac{d r_{j}}{r_{j}}= \\
& =\int_{r_{0}^{1}}^{1} \cdots \int_{r_{0}^{1}}^{1}\left(\ln r_{2}^{*}-\ln r_{1}^{*}\right)^{p-l} \prod_{i \in I} \frac{d r_{i}}{1-r_{i}}= \\
& =\int_{r_{0}^{1}}^{1} \ldots \int_{r_{0}^{1}}^{1}\left(\ln g_{2}^{-1}\left(2 g_{1}\left(r_{1}\right)\right)-\ln g_{2}^{-1}\left(\frac{g_{1}\left(r_{1}\right)}{2}\right)\right) \prod_{i \in I} \frac{d r_{i}}{1-r_{i}} \geq \\
& \geq \int_{r_{0}^{1}}^{1} \ldots \int_{r_{0}^{1}}^{1}\left(\ln \left(2 g_{1}\left(r_{1}\right)\right)-\ln \left(\frac{1}{2} \frac{3 g_{1}\left(r_{1}\right)}{2}\right)\right)^{p-l} \prod_{i \in I} \frac{d r_{i}}{1-r_{i}}= \\
& =\int_{r_{0}^{1}}^{1} \ldots \int_{r_{0}^{1}}^{1} \ln ^{p-l} \frac{8}{3} \prod_{i \in I} \frac{d r_{i}}{1-r_{i}}=+\infty .
\end{aligned}
$$

Proposition 1. For any $\varepsilon>0$ and for a function $f \in \mathcal{A}^{p}\left(\mathbb{T}_{l}\right)$ from the proof of Theorem 2 there exists $r_{0} \in T$ such that for all $r \in \triangle_{r_{0}}$ we have

$$
\mu_{f}(r) 2^{-l} \geq 1, \quad\left(\ln \left(\frac{2}{3} \sum_{j \in J} r_{j}\right)\right)^{\varepsilon} 2^{-l-\varepsilon} \geq 1, \quad\left(\ln \ln \mu_{f}(r)\right)^{a} \geq\left(\prod_{j \in J} \ln r_{j}\right)^{p},
$$

and the set 


$$
\begin{gathered}
E_{1}=\left\{r \in T: M_{f}(r)>\right. \\
\left.>\mu_{f}(r) \prod_{i \in I} \frac{1}{1-r_{i}} \ln ^{p / 2}\left(\mu_{f}(r) \prod_{i \in I} \frac{1}{1-r_{i}}\right)\left(\prod_{j \in J} \ln r_{j}\right)^{p / 2}\left(\ln \ln \mu_{f}(r)\right)^{-a}\right\},
\end{gathered}
$$

has asymptotically infinite logarithmic measure, where $a=p(p-l)+\varepsilon$.

Proof. Consider the function

$$
f(z)=f\left(z_{1}, \ldots, z_{p}\right)=\prod_{i \in I}\left(\sum_{n_{i}=0}^{+\infty} e^{\sqrt{n_{i}}} z_{i}^{n_{i}}\right) \prod_{j \in J}\left(\sum_{n_{j}=0}^{+\infty} \frac{z_{j}^{n_{j}}}{n_{j} !}\right)=\prod_{i \in I} \psi\left(z_{i}\right) \prod_{j \in J} \varphi\left(z_{j}\right) .
$$

Remark that $\ln \mu_{\varphi}(u) \geq \frac{2}{3} u, u \rightarrow+\infty$ (see [27]). Then there exists $r_{0} \in \mathbb{R}_{+}^{p}$ such that for all $r \in \triangle_{r_{0}}$ we have

$$
\left(\frac{1}{2} \prod_{j \in J} \ln r_{j}\right)^{a} \geq\left(\prod_{j \in J} \ln r_{j}\right)^{a-\varepsilon},\left(\ln \left(\frac{2}{3} \sum_{j \in J} r_{j}\right)\right)^{\varepsilon} 2^{-l-\varepsilon} \geq 1, \sum_{j \in J} \frac{2}{3} r_{j}>l \ln 2 .
$$

Thus, for all $r \in \triangle_{r_{0}}$ we get

$$
\ln \mu_{f}(r)=\sum_{i \in I} \ln \mu_{\psi}\left(r_{i}\right)+\sum_{j \in J} \ln \mu_{\varphi}\left(r_{j}\right) \geq \sum_{j \in J} \ln \mu_{\varphi}\left(r_{j}\right), \geq \sum_{j \in J} \frac{2}{3} r_{j}>l \ln 2 .
$$

i.e. $\mu_{f}(r) 2^{-l} \geq 1$, and

$$
\begin{gathered}
\left(\ln \ln \mu_{f}(r)\right)^{a} \geq \ln ^{a}\left(\sum_{j \in J} \frac{2}{3} r_{j}\right) \geq \prod_{j \in J}\left(\ln r_{j}+\ln \frac{2}{3}\right)^{a} \geq\left(\frac{1}{2} \prod_{j \in J} \ln r_{j}\right)^{a} \geq \\
\geq\left(\prod_{j \in J} \ln r_{j}\right)^{\frac{a-\varepsilon}{p-l}}=\left(\prod_{j \in J} \ln r_{j}\right)^{p} .
\end{gathered}
$$

Therefore, $E \cap \Delta_{r_{0}} \subset E_{1} \cap \Delta_{r_{0}}$. It implies that the set $E_{1}$ has asymptotically infinite logarithmic measure.

4. Wiman's inequality for random analytic functions in $\mathbb{T}=\mathbb{T}_{l}$ and analytic functions with rapidly oscillating coefficients. Let $\Omega=[0,1]$ and $P$ be the Lebesgue measure on $\mathbb{R}$. We consider the Steinhaus probability space $(\Omega, \mathcal{A}, P)$, where $\mathcal{A}$ is the $\sigma$-algebra of Lebesgue measurable subsets of $\Omega$.

Let $X=\left(X_{n}(t)\right)_{\mathbb{Z}_{+}^{p}}$ be a real multiplicative system (MS) of random variables uniformly bounded by the number 1 . That is for all $n \in \mathbb{Z}_{+}^{p}$ and $t \in[0,1]$ we have $\left|X_{n}(t)\right| \leq 1$ for almost all $t \in[0 ; 1]$ and

$$
(\forall k \in \mathbb{N})\left(\forall\left\{i_{1}, i_{2}, \ldots, i_{k}: i_{s} \neq i_{l}(s \neq l)\right\} \subset \mathbb{Z}_{+}^{p}\right): \mathbf{E}\left(X_{i_{1}} X_{i_{2}} \cdots X_{i_{k}}\right)=0,
$$

where $\mathbf{E} \xi$ is the expectation of a random variable $\xi$.

Let $Z=\left(Z_{n}(t)\right)$ be a sequence of random complex variables $Z_{n}(t)=X_{n}(t)+i Y_{n}(t)$ such that both $X=X_{n}(t)$ and $Y=Y_{n}(t)$ are real MS. 
Let $Z=\left(Z_{n}(t)\right)$ be some sequence of complex-valued random variables defined in the space $(\Omega, \mathcal{A}, P)$. For an analytic function $f$ of the form $(3)$ by $\mathcal{K}(f, Z)$ we denote the class of random analytic functions of the form

$$
f(z, t)=\sum_{\|n\|=0}^{+\infty} a_{n} Z_{n}(t) z^{n}
$$

In the sequel, the notion "almost surely" (a.s.) will be used in the sense that the corresponding property holds almost everywhere with respect to the Lebesgue measure $P$ on $\Omega$. We say that some relation holds almost surely in the class $\mathcal{K}(f, Z)$ if it holds for each analytic function $f(z, t)$ of the form (15) almost surely in $t$.

In the case when $Z=\left(X_{n}(t)\right)$ is the Rademacher sequence, under additional assumptions about the entire function $f$ P. Levy $([30])$ proved that in the classical Wiman's inequality a.s. in the class $K(f, Z)$ we can replace the constant $1 / 2$ by $1 / 4$. In the case when $Z=$ $\left(X_{n}(t)\right)$ is the Rademacher sequence P. Levy ([30]) proved that for any entire function we can replace the constant $1 / 2$ by $1 / 4$ in the Wiman's inequality a. s. in the class $K(f, Z)$ (Levy's phenomenon). P. Erdôs and A. Rényi ([9]) proved the same result for the class $K(f, H)$, where $H=\left(e^{2 \pi i \omega_{n}(t)}\right),\left(\omega_{n}(t)\right)$ is a sequence of independent uniformly distributed random variables on $[0,1]$. This statement is true also for any class $K(f, X)$, where $X=\left(X_{n}(t)\right)$ is a MS uniformly bounded by the number 1 ([31, 33, 34]).

In the spring of 1996 during the report of P. V. Filevych on the Lviv seminar of the theory of analytic functions professors A. A. Goldberg and M. M. Sheremeta posed the following question (see [21]). Does Levy's fenomenon take place for analogues of Wiman's inequality for entire functions of several complex variables?

In the papers $[18,19]$ one can find an affirmative answer to this question on Fenton's inequality ([15]) for random entire functions of two complex variables, in [23] about an inequality from [14] for random entire functions of several complex variables, in [24] in the case of analytic functions in the polydisc.

4.1. Random analytic functions in $\mathcal{A}^{p}\left(\mathbb{T}_{l}\right)$. Consider the class of random analytic functions $\mathcal{K}(f, Z)$ of the form (15) for an analytic function $f \in \mathcal{A}^{p}\left(\mathbb{T}_{l}\right)$ of the form (3) and a MS $Z=\left(Z_{n}(t)\right)$. For $r=\left(r_{1}, \ldots, r_{p}\right)$ and a function $f(z, t)$ we denote $M_{f}(r, t):=$ $\max \left\{|f(z, t)|:\left|z_{1}\right|=r_{1}, \ldots,\left|z_{p}\right|=r_{p}\right\}$.

We prove the following statement.

Theorem 3. Let $f \in \mathcal{A}^{p}, Z$ be a $M S$ uniformly bounded by the number $1, \delta>0$. Then almost surely in $t$ there exists a set $E=E(f, t, \delta), E \subset \Upsilon$ such that for all $r \in T \backslash E$ we have

$$
M_{f}(r, t) \leq \mu_{f}(r) \prod_{i \in I} \frac{1}{\left(1-r_{i}\right)^{1 / 2+\delta}} \ln ^{p / 4+\delta}\left(\mu_{f}(r) \prod_{i \in I} \frac{1}{1-r_{i}}\right)\left(\prod_{j \in J} \ln r_{j}\right)^{p / 2+\delta} .
$$

To prove the Theorem 3 we need the following lemmas.

Lemma 4 ([22]). Let $X=\left(X_{n}(t)\right)$ be a $M S$ uniformly bounded by the number 1 . Then for all $\beta>0$ there exists a constant $A_{\beta p}>0$, which depends on $p$ and $\beta$ only such that for all $N \geq N_{1}(p)=\max \{p, 4 \pi\}$ and $\left\{c_{n}:\|n\| \leq N\right\} \subset \mathbb{C}$ we have

$$
P\left\{t: \max \left\{\left|\sum_{\|n\|=0}^{N} c_{n} X_{n}(t) e^{i(n, \psi)}\right|: \psi \in[0,2 \pi]^{p}\right\} \geq A_{\beta p} S_{N} \ln ^{\frac{1}{2}} N\right\} \leq \frac{1}{N^{\beta}}
$$


where

$$
(n, \psi)=\sum_{s=1}^{p} i n_{s} \psi_{s}, S_{N}^{2}=\sum_{\|n\|=0}^{N}\left|c_{n}\right|^{2}
$$

Lemma 5. Let $\delta>0$. There exits a set $E \in \Upsilon$ such that for all $r \in T \backslash E$ we have

$$
\sum_{\|n\|=0}^{+\infty}\|n\|\left|a_{n}\right| r^{n} \leq \mu_{f}(r) \prod_{i \in I} \frac{1}{\left(1-r_{i}\right)^{2+\delta}} \ln ^{p / 2+1+\delta}\left(\mu_{f}(r) \prod_{i \in I} \frac{1}{1-r_{i}}\right) \prod_{j \in J}\left(\ln r_{j}\right)^{p+1+\delta}
$$

Proof of Lemma 5. We remark

$$
r_{i} \frac{\partial}{\partial r_{i}} \ln \mathfrak{M}_{f}(r)=\frac{1}{\mathfrak{M}_{f}(r)} \sum_{\|n\|=0}^{+\infty} n_{i}\left|a_{n}\right| r^{n}
$$

By Lemma 3, there exists a set $E \in \Upsilon$ such that for all $r \in T \backslash E_{1}$ we have

$$
\begin{gathered}
\sum_{\|n\|=0}^{+\infty}\|n\|\left|a_{n}\right| r^{n}=\mathfrak{M}_{f}(r) \sum_{i=1}^{p} r_{i} \frac{\partial}{\partial r_{i}} \ln \mathfrak{M}_{f}(r) \leq \\
\leq\left(\sum_{m \in B}\left[\frac{r_{m}}{1-r_{m}} \prod_{i \in I, i \neq m} \frac{1}{\left(1-r_{i}\right)^{\delta}}\left(\ln \mathfrak{M}_{f}(r) \prod_{j \in J} \ln r_{j}\right)^{1+\delta}\right]+\right. \\
\left.+\sum_{k \in D}\left[\prod_{i \in I} \frac{1}{\left(1-r_{i}\right)^{\delta}}\left(\ln \mathfrak{M}_{f}(r) \prod_{j \in J, j \neq k} \ln r_{j}\right)^{1+\delta}\right]\right) \leq \\
\leq \mathfrak{M}_{f}(r) \prod_{i \in I} \frac{1}{\left(1-r_{i}\right)^{1+\delta}}\left(\ln \mathfrak{M}_{f}(r)\right)^{1+2 \delta}\left(\prod_{j \in J} \ln r_{j}\right)^{1+2 \delta} .
\end{gathered}
$$

Therefore, by Theorem 1 we obtain for all $r \in T \backslash E_{2}$

$$
\begin{gathered}
\sum_{\|n\|=0}^{+\infty}\|n\|\left|a_{n}\right| r^{n} \leq 2 \mu_{f}(r) \prod_{i \in I} \frac{1}{\left(1-r_{i}\right)^{2+\delta}} \ln ^{p / 2+\delta}\left(\mu_{f}(r) \prod_{i \in I} \frac{1}{1-r_{i}}\right)\left(\prod_{j \in J} \ln r_{j}\right)^{p+\delta} \times \\
\times\left(\ln \mu_{f}(r)+(1+\delta) \sum_{i \in I} \ln \frac{1}{1-r_{i}}(p+\delta) \sum_{j \in J} \ln \ln r_{j}\right)^{1+2 \delta} \prod_{j \in J}\left(\ln r_{j}\right)^{1+2 \delta} \leq \\
\leq 2 \mu_{f}(r) \prod_{i \in I} \frac{1}{\left(1-r_{i}\right)^{2+2 \delta}} \ln ^{p / 2+1+\delta}\left(\mu_{f}(r) \prod_{i \in I} \frac{1}{1-r_{i}}\right) \prod_{j \in J}\left(\ln r_{j}\right)^{p+1+\delta} .
\end{gathered}
$$

Proof of Theorem 3. Without loss of generality we may suppose that $Z=X=\left(X_{n m}(t)\right)$ is a real MS. For $k, m \in \mathbb{Z}_{+}$and $l \in \mathbb{Z}$ such that $k>-l$ we denote

$$
\begin{aligned}
& G_{k l}=\left\{r=\left(r_{1}, r_{2}\right) \in T: k \leq \sum_{i \in I} \ln \frac{1}{1-r_{i}} \leq k+1, l \leq \ln \mu_{f}(r) \leq l+1\right\}, \\
& G_{k l m}=\left\{r=\left(r_{1}, r_{2}\right) \in G_{k l}: m \leq \sum_{j \in J} \ln \ln r_{j} \leq m+1\right\}, \quad G_{k l}^{+}=\bigcup_{i=k}^{+\infty} \bigcup_{j=l}^{+\infty} G_{i j} .
\end{aligned}
$$


Remark that

$$
E_{0}=\left\{r \in T: \sum_{i \in I} \ln \frac{1}{1-r_{i}}+\ln \mu_{f}(r)<1\right\}=\left\{r \in T: \mu_{f}(r) \prod_{i \in I} \frac{1}{1-r_{i}}<e\right\} \in \Upsilon
$$

because there exists $r_{0}$ such that $E_{0} \cap \Delta_{r_{0}}=\varnothing$.

By Lemma 5 there exists a set $E_{1} \supset E_{0}, E_{1} \in \Upsilon$ such that for all $r \in T \backslash E_{1}$ we have

$$
\begin{gathered}
\sum_{\|n\| \geq d}\left|a_{n}\right| r^{n} \leq \sum_{\|n\| \geq d} \frac{\|n\|}{d}\left|a_{n}\right| r^{n} \leq \frac{1}{d} \sum_{\|n\|=0}^{+\infty}\|n\|\left|a_{n}\right| r^{n} \leq \\
\leq \frac{1}{d} \mu_{f}(r) \prod_{i \in I} \frac{1}{\left(1-r_{i}\right)^{2+\delta}} \ln ^{p / 2+1+\delta}\left(\mu_{f}(r) \prod_{i \in I} \frac{1}{1-r_{i}}\right) \prod_{j \in J}\left(\ln r_{j}\right)^{p+1+\delta} \leq \mu_{f}(r),
\end{gathered}
$$

where

$$
d=d(r)=\prod_{i \in I} \frac{1}{\left(1-r_{i}\right)^{2+\delta}} \ln ^{p / 2+1+\delta}\left(\mu_{f}(r) \prod_{i \in I} \frac{1}{1-r_{i}}\right) \prod_{j \in J}\left(\ln r_{j}\right)^{p+1+\delta} .
$$

Let $G_{k l}^{*}=G_{k l} \backslash E_{2}, I=\left\{(i ; j): G_{i j}^{*} \neq \varnothing\right\}$,

$$
E_{2}=E_{0} \cup E_{1} \cup\left(\bigcup_{(i, j) \notin I} G_{i j}\right) .
$$

Then $\# I=+\infty$. For $(k, l) \in I$ we choose a sequence $r^{(k, l)} \in G_{k l}^{*}$ such that

$$
M_{f}\left(r^{(k, l)}\right)=\min \left\{M_{f}(r): r \in G_{k l}^{*}\right\} .
$$

So, for all $r \in G_{k l}^{*}$ we get

$$
\begin{gathered}
\frac{1}{e} \mu_{f}\left(r^{(k, l)}\right) \leq \mu_{f}(r) \leq e \mu_{f}\left(r^{(k, l)}\right), \\
\frac{1}{e} \prod_{i \in I} \frac{1}{1-r_{i}^{(k, l)}} \leq \prod_{i \in I} \frac{1}{1-r_{i}} \leq e \prod_{i \in I} \frac{1}{1-r_{i}^{(k, l)}}, \\
\frac{1}{e^{2}} \mu_{f}\left(r^{(k, l)}\right) \prod_{i \in I} \frac{1}{1-r_{i}^{(k, l)}} \leq \mu_{f}(r) \prod_{i \in I} \frac{1}{1-r_{i}} \leq e^{2} \mu_{f}\left(r^{(k, l)}\right) \prod_{i \in I} \frac{1}{1-r_{i}^{(k, l)}}
\end{gathered}
$$

and also

$$
\bigcup_{(k, l) \in I} G_{k l}^{*}=\bigcup_{(k, l) \in I} G_{k l} \backslash E_{2}=\bigcup_{k, l=1}^{+\infty} G_{k l} \backslash E_{2}=T \backslash E_{2}
$$

Denote $N_{k l}=\left[2 d_{1}\left(r^{(k, l)}\right)\right]$, where

$$
d_{1}(r)=e^{2+\delta} \prod_{i \in I} \frac{1}{\left(1-r_{i}\right)^{2+\delta}} \ln ^{p / 2+1+\delta}\left(e^{2} \mu_{f}(r) \prod_{i \in I} \frac{1}{1-r_{i}}\right) \prod_{j \in J}\left(\ln \left(e r_{j}\right)\right)^{p+1+\delta} .
$$

For $r \in G_{k l}^{*}$ we put

$$
W_{N_{k l}}(r, t)=\max \left\{\left|\sum_{\|n\| \leq N_{k l}} a_{n} r^{n} X_{n}(t) e^{i(n, \psi)}\right|: \psi \in[0,2 \pi]^{p}\right\} .
$$


For a Lebesgue measurable set $G \subset G_{k l}^{*}$ and for $(k, l) \in I$ we denote

$$
\nu_{k l}(G)=\frac{\operatorname{meas}(G)}{\operatorname{meas}\left(G_{k l}^{*}\right)}
$$

where meas denotes the Lebesgue measure on $\mathbb{R}^{p}$.

Remark that $\nu_{k l}$ is a probability measure defined on the $\sigma$-algebra of Lebesgue measurable subsets of $G_{k}^{*}([23])$. Let

$$
\Omega=\bigcup_{(k, l) \in I} G_{k l}^{*}
$$

and

$$
k_{i}, l_{i, j}:\left(k_{i}, l_{i, j}\right) \in I, k_{i}<k_{i+1}, l_{i, j}<l_{i, j+1}, \forall i, j \in \mathbb{Z}_{+} .
$$

For Lebesgue measurable subsets $G$ of $\Omega$ we denote

$$
\begin{gathered}
\nu(G)=2^{k_{0}} \sum_{i=0}^{+\infty}\left(\frac{1}{2^{k_{i}}}\left(1-\left(\frac{1}{2}\right)^{k_{i+1}-k_{i}}\right) \times\right. \\
\left.\times \sum_{j=0}^{N_{i}} \frac{2^{l_{i, 0}}}{2^{l_{i, j}}} \frac{\left(1-\left(\frac{1}{2}\right)^{l_{i, j+1}-l_{i, j}}\right)}{1-\left(\frac{1}{2}\right)^{l_{i, N_{i+1}}+l_{i, 0}}} \nu_{k_{i+1} l_{i+1, j+1}}\left(G \cap G_{k_{j+1} l_{i+1, j+1}}^{*}\right)\right),
\end{gathered}
$$

where $N_{i}=\max \left\{j:\left(k_{i}, l_{i j}\right) \in I\right\}$. It easy to se that $\nu_{k_{j+1} l_{j+1}}\left(G_{k_{j+1} l_{j+1}}^{*}\right)=\nu(\Omega)=1$.

Thus $\nu$ is a probability measure, which is defined on measurable subsets of $\Omega$. On $[0,1] \times \Omega$ we define the probability measure $P_{0}=P \otimes \nu$, which is the direct product of the probability measures $P$ and $\nu$. Now for $(k ; l) \in I$ we define

$$
\begin{gathered}
F_{k l}=\left\{(t, r) \in[0,1] \times \Omega: W_{N_{k l}}(r, t)>A S_{N_{k l}}(r) \ln ^{1 / 2} N_{k l}\right\}, \\
F_{k l}(r)=\left\{t \in[0,1]: W_{N_{k l}}(r, t)>A S_{N_{k l}}(r) \ln ^{1 / 2} N_{k l}\right\},
\end{gathered}
$$

where

$$
S_{N_{k l}}^{2}(r)=\sum_{\|n\|=0}^{N_{k l}}\left|a_{n}\right|^{2} r^{2 n}
$$

and $A$ is the constant from Lemma 4 with $\beta=1$. Using Fubini's theorem and Lemma 4 with $c_{n}=a_{n} r^{n}$ and $\beta=1$, we get for $(k, l) \in I$

$$
P_{0}\left(F_{k l}\right)=\int_{\Omega}\left(\int_{F_{k l}(r)} d P\right) d \nu=\int_{\Omega} P\left(F_{k l}(r)\right) d \nu \leq \frac{1}{N_{k l}} \nu(\Omega)=\frac{1}{N_{k l}} .
$$

Note that

$$
N_{k l}>\prod_{i \in I} \frac{1}{\left(1-r_{i}^{(k, l)}\right)^{2+\delta}} \ln ^{p / 2+1+\delta}\left(\mu_{f}\left(r^{(k, l)}\right) \prod_{i \in I} \frac{1}{1-r_{i}^{(k, l)}}\right) \prod_{j \in J}\left(\ln r_{j}^{(k, l)}\right)^{p+1+\delta} \geq e^{2 k}(l+k)^{2+\delta} .
$$

Therefore,

$$
\sum_{(k, l) \in I} P_{0}\left(F_{k l}\right) \leq \sum_{k=1}^{+\infty} \sum_{l=-k+1}^{+\infty} \frac{1}{e^{2 k}(l+k)^{2+\delta}}<+\infty
$$


By Borel-Cantelli's lemma the infinite quantity of the events $\left\{F_{k l}:(k, l) \in I\right\}$ may occur with probability zero. So,

$$
P_{0}(F)=1, \quad F=\bigcup_{s=1}^{+\infty} \bigcup_{m=1}^{+\infty} \bigcap_{\substack{k \geq s, l \geq m \\(k, l) \in I}} \overline{F_{k l}} \subset[0,1] \times \Omega .
$$

Then for any point $(t, r) \in F$ there exist $k_{0}=k_{0}(t, r)$ and $l_{0}=l_{0}(t, r)$ such that for all $k \geq k_{0}, l \geq l_{0},(k, l) \in I$ we have

$$
W_{N_{k l}}(r, t) \leq A S_{N_{k l}}(r) \ln ^{1 / 2} N_{k l} .
$$

So, $\nu\left(F^{\wedge}(t)\right)=1$ (see [23]).

For any $t \in F_{1}([23])$ and $(k, l) \in I$ we choose a point $r_{0}^{(k, l)}(t) \in G_{k l}^{*}$ such that

$$
W_{N_{k l}}\left(r_{0}^{(k, l)}(t), t\right) \geq \frac{3}{4} M_{k l}(t), M_{k l}(t) \stackrel{\text { def }}{=} \sup \left\{W_{N_{k l}}(r, t): r \in G_{k l}^{*}\right\} .
$$

Then from $\nu_{k l}\left(F^{\wedge}(t) \cap G_{k l}^{*}\right)=1$ for all $(k, l) \in I$ it follows that there exists a point $r^{(k, l)}(t) \in G_{k l}^{*} \cap F^{\wedge}(t)$ such that

$$
\left|W_{N_{k l}}\left(r_{0}^{(k, l)}(t), t\right)-W_{N_{k l}}\left(r^{(k, l)}(t), t\right)\right|<\frac{1}{4} M_{k l}(t),
$$

hence

$$
W_{N_{k l}}\left(r^{(k, l)}(t), t\right)-W_{N_{k l}}\left(r_{0}^{(k, l)}(t), t\right)<\frac{1}{4} M_{k l}(t)
$$

Thus,

$$
\frac{3}{4} M_{k l}(t) \leq W_{N_{k l}}\left(r_{0}^{(k, l)}(t), t\right) \leq W_{N_{k l}}\left(r^{(k, l)}(t), t\right)+\frac{1}{4} M_{k l}(t) .
$$

Since $\left(t, r^{(k, l)}(t)\right) \in F$, we obtain

$$
\frac{1}{2} M_{k l}(t) \leq W_{N_{k l}}\left(r^{(k, l)}(t), t\right) \leq A S_{N_{k l}}\left(r^{(k, l)}(t)\right) \ln ^{1 / 2} N_{k l} .
$$

Now for $r^{(k, l)}=r^{(k, l)}(t)$ we get

$$
\begin{gathered}
S_{N_{k l}}^{2}\left(r^{(k, l)}\right) \leq \mu_{f}\left(r^{(k, l)}\right) \mathfrak{M}_{f}\left(r^{(k, l)}\right) \leq \\
\leq \mu_{f}^{2}\left(r^{(k, l)}\right) \prod_{i \in I} \frac{1}{\left(1-r_{i}^{(k, l)}\right)^{1+\delta}} \ln ^{p / 2+\delta}\left(\mu_{f}\left(r^{(k, l)}\right) \prod_{i \in I} \frac{1}{1-r_{i}^{(k, l)}}\right)\left(\prod_{j \in J} \ln r_{j}^{(k, l)}\right)^{p+\delta} .
\end{gathered}
$$

So, for $t \in F_{1}$ and all $k \geq k_{0}(t), l \geq l_{0}(t)$, we obtain

$$
\begin{gathered}
S_{N_{k l}\left(r^{(k, l)}\right)} \leq \\
\leq \mu_{f}\left(r^{(k, l)}\right) \prod_{i \in I} \frac{1}{\left(1-r_{i}^{(k, l)}\right)^{1 / 2+\delta}} \ln ^{p / 4+\delta}\left(\mu_{f}\left(r^{(k, l)}\right) \prod_{i \in I} \frac{1}{1-r_{i}^{(k, l)}}\right)\left(\prod_{j \in J} \ln r_{j}^{(k, l)}\right)^{p / 2+\delta} .
\end{gathered}
$$

It follows from (19)-(21) that $d_{1}\left(r^{(k, l)}\right) \geq d(r)$ for $r \in G_{k l}^{*}$. Then for $t \in F_{1}, r \in F^{\wedge}(t) \cap G_{k l}^{*}$, $(k, l) \in I, k \geq k_{0}(t), l \geq l_{0}(t)$ we get

$$
M_{f}(r, t) \leq \sum_{\|n\| \geq 2 d_{1}\left(r^{(k, l)}\right)}\left|a_{n}\right| r^{n}+W_{N_{k l}}(r, t) \leq \sum_{\|n\| \geq 2 d(r)}\left|a_{n}\right| r^{n}+M_{k l}(t) .
$$


Finally, for $t \in F_{1}, r \in F^{\wedge}(t) \cap G_{k l}^{*}, l \geq l_{0}(t)$ and $k \geq k_{0}(t)$ we deduce

$$
\begin{gathered}
M_{f}\left(r^{(k, l)}, t\right) \leq \mu_{f}\left(r^{(k, l)}\right)+2 A S_{N_{k l}}\left(r^{(k, l)}\right) \ln ^{1 / 2} N_{k l} \leq \mu_{f}\left(r^{(k, l)}\right)+ \\
+2 A \mu_{f}\left(r^{(k, l)}\right) \prod_{i \in I} \frac{1}{\left(1-r_{i}^{(k, l)}\right)^{1 / 2+\delta}} \ln ^{p / 4+\delta}\left(\mu_{f}\left(r^{(k, l)}\right) \prod_{i \in I} \frac{1}{1-r_{i}^{(k, l)}}\right)\left(\prod_{j \in J} \ln r_{j}^{(k, l)}\right)^{p / 2+\delta} \times \\
\left.\times \ln \left(e^{2+\delta} \prod_{i \in I} \frac{1}{\left(1-r_{i}^{(k, l)}\right)^{2+\delta}} \ln ^{p / 2+1+\delta}\left(e^{2} \mu_{f}\left(r^{(k, l)}\right) \prod_{i \in I} \frac{1}{1-r_{i}^{(k, l)}}\right) \prod_{j \in J}\left(\ln \left(e r_{j}^{(k, l)}\right)\right)^{p+1+\delta}\right)\right) .
\end{gathered}
$$

Therefore the following inequality

$$
M_{f}(r, t) \leq \mu_{f}(r) \prod_{i \in I} \frac{1}{\left(1-r_{i}\right)^{1 / 2+2 \delta}} \ln ^{p / 4+2 \delta}\left(\mu_{f}(r) \prod_{i \in I} \frac{1}{1-r_{i}}\right)\left(\prod_{j \in J} \ln r_{j}\right)^{p / 2+2 \delta} .
$$

holds a.s. $\left(t \in F_{1}, P\left(F_{1}\right)=1\right)$ for all

$$
r \in\left(\bigcup_{(k, l) \in I}\left(G_{k l}^{*} \cap F^{\wedge}(t)\right) \cap G_{k l}^{+}\right) \backslash E^{*}=\left(T \cap G_{k l}^{+}\right) \backslash\left(E^{*} \cup G^{*} \cup E_{1}\right)=T \backslash E_{2},
$$

where

$$
G_{k l}^{+}=\bigcup_{i=k}^{+\infty} \bigcup_{j=l}^{+\infty} G_{k l}, E_{2}=E_{1} \cup G^{*} \cup E^{*}, G^{*}=\bigcup_{(k, l) \in I}\left(G_{k l}^{*} \backslash F^{\wedge}(t)\right) .
$$

It remains to remark that $\nu\left(G^{*}\right)$ satisfies

$$
\nu\left(G^{*}\right)=\sum_{(k, l) \in I}\left(\nu_{k l}\left(G_{k l}^{*}\right)-\nu_{k l}\left(F^{\wedge}(t)\right)\right)=0
$$

Then for all $(k, l) \in I$ we obtain

$$
\begin{gathered}
\nu_{k l}\left(G_{k l}^{*} \backslash F^{\wedge}(t)\right)=\frac{\operatorname{meas}\left(G_{k l}^{*} \backslash F^{\wedge}(t)\right)}{\operatorname{meas}\left(G_{k l}^{*}\right)}=0, \\
\operatorname{meas}\left(G_{k l}^{*} \backslash F^{\wedge}(t)\right)=\int_{G_{k l}^{*} \backslash F^{\wedge}(t)} \ldots \int \prod_{i \in I} \frac{d r_{i}}{1-r_{i}} \prod_{j \in J} \frac{d r_{j}}{r_{j}}=0 .
\end{gathered}
$$

4.2. Analytic functions with rapidly oscillating coefficients. We consider the class $\mathcal{K}(f, \theta)$ of analytic functions

$$
f(z, t)=f\left(z_{1}, \ldots, z_{p}, t\right)=\sum_{\|n\|=0}^{+\infty} a_{n} e^{2 \pi i \theta_{n} t} z^{n}
$$

Here $\theta=\left(\theta_{n}\right)$ is a sequence of positive integer such that its increasing arrangement $\left(\theta_{k}^{*}\right)$ $\left\{\theta_{n}: n \in \mathbb{Z}_{+}^{p}\right\}=\left\{\theta_{k}^{*}: k \in \mathbb{Z}_{+}\right\}, \theta_{k+1}^{*}>\theta_{k}^{*}$, satisfies the condition ( $\theta$ is Hadamard sequence)

$$
\theta_{k+1}^{*} / \theta_{k}^{*} \geq q>1, k>0
$$


where $f \in \mathcal{A}^{p}\left(\mathbb{T}_{l}\right)$. Remark, that in the case $q \geq 2$ analytic functions of the form (24) satisfy the assumptions of Theorem 3 , because $\left(\cos \theta_{n} t\right),\left(\sin \theta_{n} t\right)$ are MS. But in the case $q>1$ the sequence of random variables $\left(\cos \theta_{n} t\right)_{n \in \mathbb{Z}_{+}^{\prime}}$ need not be a MS (see [21]). So the following question arrives naturally: does Levy's phenomenon hold for the class $\mathcal{K}(f, \theta)$ with $f \in \mathcal{A}^{p}$ and an Hadamard sequence $\theta$ ?

A theorem analogous to Theorem 3 is also valid for analytic functions with rapidly oscillating coefficients.

Theorem 4. Let $\delta>0, f \in \mathcal{K}(f, \theta)$ be an analytic function of the from (24) and a sequence of positive integers $\left(\theta_{n}\right)_{n \in \mathbb{Z}_{+}^{p}}$ satisfy condition (25). Then almost surely for $t \in \mathbb{R}$ there exists $E(\delta, t) \in \Upsilon$ such that for all $r \in T \backslash E$ we have

$$
M_{f}(r, t) \leq \mu_{f}(r) \prod_{i \in I} \frac{1}{\left(1-r_{i}\right)^{1 / 2+\delta}} \ln ^{p / 4+\delta}\left(\mu_{f}(r) \prod_{i \in I} \frac{1}{1-r_{i}}\right)\left(\prod_{j \in J} \ln r_{j}\right)^{(p-l) / 4+\delta} .
$$

One can find similar inequalities for entire functions of one complex variable in $[9,10]$, for analytic functions in the unit disc in [13], for entire functions of several variables in [18], [19], [23], [26], [29], and for analytic functions without exceptional sets in [16, 32].

To prove Theorem 4 we need an auxiliary assertions.

Lemma 6 ([10]). Let $\left(\theta_{k}^{*}\right)_{k=1}^{N}$ be a sequence of integers such that condition (25) holds. Then there exist constants $A_{q}$ and $B_{q}$ (depending only on $q$ ) such that for any $\left\{b_{k}: 1 \leq k \leq N\right\} \subset \mathbb{C}$ and $\lambda>0$ we have

$$
P\left\{t:\left|\sum_{k=1}^{N} b_{k} e^{2 \pi i \theta_{k}^{*} t}\right| \geq A_{q} \lambda S_{N}\right\} \leq B_{q} e^{-\lambda^{2}},
$$

where $S_{N}^{2}=\sum_{k=1}^{n}\left|b_{k}\right|^{2}, P$ is the Lebesque measure on $[0 ; 1]$.

Lemma 7 ([28]). Let $\theta=\left(\theta_{n}\right)_{n \in \mathbb{Z}_{+}^{p}}$ be a sequence of integers satisfying (25). Then for any $\beta>0, p \geq 1, l \in \mathbb{N}, l \geq p$ and $\left\{c_{n}: n \in \mathbb{Z}_{+}^{p}\right\} \subset \mathbb{C}$ we get

$$
P\left\{t: \max \left\{\left|\sum_{\|n\|=0}^{l} c_{n} \exp \left\{i(n, \psi)+2 \pi i \theta_{n} t\right\}\right|: \psi \in[0,2 \pi]^{p}\right\} \geq A_{\beta p} S_{l} \ln ^{1 / 2} l\right\} \leq \frac{(5 \pi+1)^{p} B}{l^{\beta}}
$$

where

$$
(n, \psi)=\sum_{s=1}^{p} n_{s} \psi_{s}, S_{l}^{2}=\sum_{\|n\|=0}^{l}\left|c_{n}\right|^{2}, A=\sqrt{\beta+\frac{p}{2}(3+p)} A_{q}+1
$$

and $B=B_{q}\left(A_{q}, B_{q}\right.$ are constants from Lemma 6$)$.

Proof of Theorem 4. To prove Theorem 4, we need to rewrite formally the proof of Theorem 3, using Lemma 7 instead of Lemma 5. We need to use Lemma 5 and Theorem 1 again. The only formal difference is that in the proof of Theorem 3 we need to change the definition $W_{N_{k l}}(r, t)$ onto

$$
W_{N_{k l}}(r, t)=\max \left\{\left|\sum_{\|n\| \leq N_{k l}} a_{n} r^{n} e^{i(n, \psi)+2 \pi i \theta_{n} t}\right|: \psi \in[0,2 \pi]^{p}\right\} .
$$

Retaining all the other notations from the proof of Theorem 3 and literally rewriting this proof, we obtain the assertion of Theorem 4. 
5. Sharpness of Theorems 3 and 4 . We will prove that any of exponents $p / 4+\delta$ and $1 / 2+\delta$ in inequality (5) cannot be replaced by a number smaller than $p / 4$ and $1 / 2$, respectively.

Theorem 5. Let $1 \leq l<p$ and $Z=\left(Z_{n}(t)\right)$ be a sequence of random variables such that $(\forall n):\left|Z_{n}(t)\right| \geq 1$ a.s. in $[0 ; 1]$. Then for any $\varepsilon>0$ there exist an analytic function $f \in \mathcal{A}^{p}\left(\mathbb{T}_{l}\right)$, a constant $C>0$ and $r_{0} \in T$ such that a.s. in $t$ for all $r \in \Delta_{r_{0}}$ we get

$$
M_{f}(r, t) \geq \mu_{f}(r) \prod_{i \in I} \frac{1}{\sqrt{1-r_{i}}} \ln ^{p / 4}\left(\mu_{f}(r) \prod_{i \in I} \frac{1}{1-r_{i}}\right)\left(\prod_{j \in J} \ln r_{j}\right)^{p / 2}\left(\ln \ln \mu_{f}(r)\right)^{-p(p-l) / 2-\varepsilon} .
$$

Proof. Consider the functions

$$
\begin{gathered}
g(z)=\prod_{i \in I}\left(\sum_{n_{i}=0}^{+\infty} e^{\sqrt{n_{i}}} z_{i}^{n_{i}}\right) \prod_{j \in J}\left(\sum_{n_{j}=0}^{+\infty} \frac{z_{j}^{n_{j}}}{n_{j} !}\right)=\sum_{\|n\|=0}^{+\infty} g_{n} z^{n} \\
f(z)=\prod_{i \in I}\left(\sum_{n_{i}=0}^{+\infty} e^{\sqrt{n_{i}} / 2} z_{i}^{n_{i}}\right) \prod_{i \in I}\left(\sum_{n_{j}=0}^{+\infty} \frac{z_{j}^{n_{j}}}{\sqrt{n_{j} !}}\right)=\sum_{\|n\|=0}^{+\infty} f_{n} z^{n} \\
f(z, t)=\sum_{\|n\|=0}^{+\infty} Z_{n}(t) f_{n} z^{n} .
\end{gathered}
$$

Remark, that for all $r \in T$ we have

$$
\begin{gathered}
\mu_{g}\left(r^{2}\right)=\max \left\{\prod_{i \in I} \frac{r_{i}^{2 n_{i}}}{n_{i} !} \prod_{j \in J} e^{\sqrt{n_{j}}} r_{j}^{2 n_{j}}: n \in \mathbb{Z}_{+}^{p}\right\}= \\
=\max \left\{\left(\prod_{i \in I} \frac{r_{i}^{n_{i}}}{\sqrt{n_{i} !}} \prod_{j \in J} e^{\sqrt{n_{j}} / 2} r_{j}^{n_{j}}\right)^{2}: n \in \mathbb{Z}_{+}^{p}\right\}=\left(\mu_{f}(r)\right)^{2} .
\end{gathered}
$$

Using Parseval's equality, we get for almost all $t$

$$
M_{g}\left(r^{2}\right) \leq \sum_{\|n\|=0}^{+\infty}\left|Z_{n}(t)\right|^{2}\left|g_{n}\right| r^{2 n}=\frac{1}{(2 \pi)^{p}} \int_{[0,2 \pi]^{p}} \ldots \int\left|f\left(r e^{i \theta}, t\right)\right|^{2} d \theta \leq\left(M_{f}(r, t)\right)^{2} .
$$

Then by Proposition 1 there exists $r_{0} \in T$ such that for $r \in \Delta_{r_{0}} \cup E$

$$
M_{g}(r)>\mu_{g}(r) \prod_{i \in I} \frac{1}{1-r_{i}} \ln ^{p / 2}\left(\mu_{g}(r) \prod_{i \in I} \frac{1}{1-r_{i}}\right)\left(\prod_{j \in J} \ln r_{j}\right)^{p}\left(\ln \ln \mu_{g}(r)\right)^{-a},
$$

where $a=p(p-l)+\varepsilon$.

Hence, for all $r$ such that $r^{2}=\left(r_{1}^{2}, \ldots, r_{p}^{2}\right) \in \Delta_{r_{0}} \cap E$ one has

$$
\begin{gathered}
\left(M_{f}(r, t)\right)^{2} \geq M_{g}\left(r^{2}\right) \geq \\
\geq \mu_{g}\left(r^{2}\right) \prod_{i \in I} \frac{1}{1-r_{i}^{2}} \ln ^{p / 2}\left(\mu_{g}\left(r^{2}\right) \prod_{i \in I} \frac{1}{1-r_{i}^{2}}\right)\left(\prod_{j \in J} \ln r_{j}^{2}\right)^{p}\left(\ln \ln \mu_{g}\left(r^{2}\right)\right)^{-a} \geq \\
\geq \mu_{f}^{2}(r) \prod_{i \in I} \frac{1}{1-r_{i}^{2}} \ln ^{p / 2}\left(\mu_{f}^{2}(r) \prod_{i \in I} \frac{1}{1-r_{i}^{2}}\right)\left(\prod_{j \in J} \ln r_{j}^{2}\right)^{p}\left(\ln \ln \mu_{f}^{2}(r)\right)^{-a} .
\end{gathered}
$$


But $1-r_{i}^{2} \leq 2\left(1-r_{i}\right)(i \in I)$, and by Proposition 1

$$
\left(\ln \ln \mu_{f}(r)\right)^{\varepsilon} \geq\left(\ln \left(\frac{2}{3} \sum_{j \in J} r_{j}\right)\right)^{\varepsilon} \geq 2^{l+\varepsilon} .
$$

Thus

$$
\begin{gathered}
\left(M_{f}(r, t)\right)^{2} \geq \\
\geq \mu_{f}^{2}(r) 2^{-l} \prod_{i \in I} \frac{1}{1-r_{i}} \ln ^{p / 2}\left(2^{-l} \mu_{f}^{2}(r) \prod_{i \in I} \frac{1}{1-r_{i}}\right)\left(2^{p-l} \prod_{j \in J} \ln r_{j}\right)^{p}\left(\ln \ln \mu_{f}^{2}(r)\right)^{-a} \geq \\
\geq 2^{p(p-l)-l-a} \mu_{f}^{2}(r) \prod_{i \in I} \frac{1}{1-r_{i}} \ln ^{p / 2}\left(\mu_{f}(r) \prod_{i \in I} \frac{1}{1-r_{i}}\right)\left(\prod_{j \in J} \ln r_{j}\right)^{p}\left(\ln \ln \mu_{f}(r)\right)^{-a} \geq \\
\geq \mu_{f}^{2}(r) \prod_{i \in I} \frac{1}{1-r_{i}} \ln ^{p / 2}\left(\mu_{f}(r) \prod_{i \in I} \frac{1}{1-r_{i}}\right)\left(\prod_{j \in J} \ln r_{j}\right)^{p}\left(\ln \ln \mu_{f}(r)\right)^{-a-\varepsilon} .
\end{gathered}
$$

Therefore, for all $r$ such that $r^{2}=\left(r_{1}^{2}, \ldots, r_{p}^{2}\right) \in \Delta_{r_{0}} \cap E$ we obtain

$$
\begin{gathered}
M_{f}(r, t) \geq \\
\geq \mu_{f}(r) \prod_{i \in I} \frac{1}{\sqrt{1-r_{i}}} \ln ^{p / 4}\left(\mu_{f}(r) \prod_{i \in I} \frac{1}{1-r_{i}}\right)\left(\prod_{j \in J} \ln r_{j}\right)^{p / 2}\left(\ln \ln \mu_{f}(r)\right)^{-a / 2-\varepsilon_{1}},
\end{gathered}
$$

where $\varepsilon_{1}=\varepsilon / 2$.

\section{REFERENCES}

1. Wiman A. Über dem Zusammenhang zwischen dem Maximalbetrage einer analytischen Funktion und dem grössten Gliede der zugehörigen Taylorschen Reihe// Acta Math. - 1914. - V.37. - P. 305-326.

2. Polya G., Szegó G. Aufgaben und Lehrsätze aus der Analysis, V.2. - Berlin, Springer, 1925.

3. Valiron G. Fonctions analytiques. - Paris: Press. Univer. de France, 1954.

4. Wittich H. Neuere Untersuchungen über eindeutige analytische Funktionen. - Berlin-GöttingenHeidelberg: Springer, 1955. - 164 s.

5. Gol'dberg A.A., Levin B.Y., Ostrovskii I.V., Entire and meromorphic functions. - Itogi Nauki i Tekhniki. Seriya VINITI. - 1990. - V.85. - P. 5-186. (in Russian)

6. Skaskiv O.B., Filevych P.V., On the size of an exceptional set in the Wiman theorem, Mat. Stud. - 1999. - V.12, №1. - P. 31-36. (in Ukrainian)

7. Skaskiv O.B., Zrum O.V. On an exeptional set in the Wiman inequalities for entire functions, Mat. Stud. - 2004. - V.21, №1. - P. 13-24. (in Ukrainian)

8. Skaskiv O.B., Bandura A.I., Asymptotic estimates of positive integrals and entire functions. - LvivIvano-Frankivsk: LNU-INFTUNG, 2015. - 108 p. (in Ukrainian)

9. Erdôs P., Rényi A. On random entire function// Zastosowania mat. - 1969. - V.10. - P. 47-55.

10. Steele J.M., Sharper Wiman inequality for entire functions with rapidly oscillating coefficients// J. Math. Anal. and Appl. - 1987. - V.123. - P. 550-558. 
11. Kövari T. On the maximum modulus and maximal term of functions analytic in the unit disc// J. London Math. Soc. - 1966. - V.41. - P. 129-137.

12. Suleymanov N.M. Wiman-Valiron's type inequalities for power series with bounded radii of convergence and its sharpness// DAN SSSR. - 1980. - V.253, №4. - P. 822-824. (in Russian)

13. Skaskiv O.B., Kuryliak A.O. Direct analogues of Wiman's inequality for analytic functions in the unit disk// Carpathian Math. Publ. - 2010. - V.2, №1. - P. 109-118. (in Ukrainian)

14. Gopala Krishna J., Nagaraja Rao I.H. Generalised inverse and probability techniques and some fundamental growth theorems in $\mathbb{C}^{k} / /$ J. Indian Math. Soc. - 1977. - V.41. - P. 203-219.

15. Fenton P.C. Wiman-Valiron theory in two variables// Trans. Amer. Math. Soc. - 1995. - V.347, №11. - P. 4403-4412.

16. Kuryliak A.O., Skaskiv O.B. Wiman's type inequalities without exceptional sets for random entire functions of several variables// Mat. Stud. - 2012. - V.38, №1. - P. 35-50.

17. Schumitzky A. Wiman-Valiron theory for functions of several complex variables. - Ph. D. Thesis: Cornel. Univ., 1965.

18. Skaskiv O.B., Zrum O.V. Wiman's type inequality for entire functions of two complex variables with rapidly oscilic coefficient// Mat. metods and fys.-mekh. polya. - 2005. - V.48, №4. - P. 78-87. (in Ukrainian)

19. Skaskiv O.B., Zrum O.V. On inprovement of Fenton's inequality for entire functions of two complex variables// Math. Bull. Shevchenko Sci. Soc. - 2006. - V.3. - P. 56-68. (in Ukrainian)

20. Skaskiv O.B., Trakalo O.M. On classical Wiman's inequality for multiple entire Dirichlet series// Mat. metods and fys.-mekh. polya. - 2000. - V.43, №3. - P. 34-39. (in Ukrainian)

21. Zrum O.V., Skaskiv O.B. On Wiman's inequality for random entire functions of two variables// Mat. Stud. - 2005. - V.23, №2. - P. 149-160. (in Ukrainian)

22. Kuryliak A.O., Skaskiv O.B. Wiman's type inequalities without exceptional sets for random entire functions of several variables// Mat. Stud. - 2012. - V.38, №1. - P. 35-50.

23. Kuryliak A.O., Skaskiv O.B., Zrum O.V. Levy's phenomenon for entire functions of several variables// Ufa Math. J. - 2014. - V.6, №2 - P.118-127.

24. Kuryliak A.O., Skaskiv O.B., Skaskiv S.R. Levy's phenomenon for analytic functions in the polydisc// ArXiv:160204756v1 [math.CV] 15 Feb 2016. - 14 p.

25. Kuryliak A.O., Shapovalovska L.O., Skaskiv O.B. Wiman's type inequality for analytic functions in the polydisc// Ukr. Mat. J. - 2016. - V.68. - P. 78-86.

26. Kuryliak A.O., Skaskiv O.B., Skaskiv S.R. Analogues of Wiman's inequality and Levy's phenomenon for analytic functions in bidisc// Buk. Mat. J. - 2015. - V.3, №3-4. - P. 102-110. (in Ukrainian)

27. Kuryliak A.O., Shapovalovska L.O., Skaskiv O.B. Wiman's type inequality for some double power series// Mat. Stud. - 2013. - V.39, №2. - P. 134-141.

28. Kuryliak A.O., Shapovalovska L.O. Wiman's type inequality for entire functions of several complex varibles with rapidly oscillating coefficients// Mat. Stud. - 2015. - V.43, №1. - P. 16-26.

29. Kuryliak A., Skaskiv O., Tsvigun V. Levy's phenomenon for analytic functions in $\mathbb{D} \times \mathbb{C} / /$ Mat. Stud. 2016. - V.46, №2. - P. 121-129.

30. Lévy P. Sur la croissance de fonctions entière // Bull. Soc. Math. France. - 1930. - V.58. - P. 29-59; P. $127-149$

31. Filevych P.V. Some classes of entire functions in which the Wiman-Valiron inequality can be almost certainly improved// Mat. Stud. - 1996. - V.6. - P. 59-66. (in Ukrainian)

32. Filevych P.V. Wiman-Valiron type inequalities for entire and random entire functions of finite logarithmic order// Sib. Mat. Zhurn. - 2003. - V.42, №3. - P. 683-694. (in Russian) English translation in: Siberian Math. J. - 2003. - V.42, №3. - P. 579-586.

33. Kuryliak A.O., Skaskiv O.B. Ineguality of Wiman' type for analytic in a disc functions and the Baire categories// Nauk. Visn. Cherniv. Natiomal Univ. Ser.: Mat. - 2011. - V.1, №4. - P.73-79. (in Ukrainian) 
34. Kuryliak A.O., Skaskiv O.B., Chyzhykov I.E. Baire categories and Wiman's inequality for analytic functions// Bull. Soc. Sc. et des letters de Lodz. - 2012. - V.62, №3. - P. 17-33.

35. Godwin H.J. On generalizations of Tchebycheff's inequality// J. Amer Stat. Assoc. - 1955. - V.50. P. 923-945.

36. Savage I.R. Probability inequalities of the Tchebycheff type// J. Research National Bureau of Stand. 1961. - V.65B, №3. - P. 211-222.

Ivan Franko National University of Lviv, Ukraine andriykuryliak@gmail.com

12lvan.n@ukr.net

Received 30.06.2017

Revised 5.01.2018 